\title{
Fully Self-Consistent RPA Description of the Many Level Pairing Model
}

\author{
Jorge G. Hirsch \\ Instituto de Ciencias Nucleares, Universidad Nacional Autónoma de México, Apdo. Postal 70-543, \\ México 04510 D.F., Mexico \\ Alejandro Mariano \\ Departamento de Física, Universidad Nacional de La Plata, C.C. 67, La Plata 1900, Argentina \\ Jorge Dukelsky \\ Instituto de Estructura de la Materia, Consejo Superior de Investigaciones Cientificas, \\ Serrano 123, 28006 Madrid, Spain \\ and \\ Peter Schuck \\ Institut de Physique Nucléaire, Université Paris-Sud, F-91406 Orsay Cedex, France
}

Received July 31, 2001

\begin{abstract}
The self-consistent RPA (SCRPA) equations in the particle-particle channel are solved without any approximation for the picket fence model. The results are in excellent agreement with the exact solutions found with the Richardson method. Particularly interesting features are that screening corrections reverse the sign of the interaction and that SCRPA yields the exact energies in the case of two levels with two particles. (1) 2002 Elsevier Science (USA)
\end{abstract}

\section{INTRODUCTION}

The approach to the many body problem is often divided into the evaluation of a hierarchy of correlation functions. First comes the one body or mean fiel theory, second the two body correlation functions, and so on. There is a large consensus on the mean fiel or Hartree-Fock level so that practically in any fiel of theoretical physics one basically understands the same by this approach in spite of the fact that there may be differences in detail. However, already on the next level of complication, i.e., considering two body correlations, the strategies in various field start to diverge strongly. Let us mention firs a few of them: Brueckner-Hartree-Fock [1], Jastrow ansatz for correlated wave functions, the hypernetted chain method, correlated basis functions [2], coupled cluster theory [3], Gutzwiller ansatz for correlated ground states [4], and quite a few more are examples of different strategies for tackling the problem of strongly correlated quantum systems. There seems to be no common consensus about which of the strategies is best; all seem to be more or less tailored for specifi problems. In this situation it seems legitimate to explore further routes which have been not sufficientl exploited in the past. It is the latter case which we have been investigating 
for a couple of years, further developing the so-called self-consistent RPA (SCRPA) theory which has been initiated mostly in nuclear physics with the pioneering work of Hara [5], and further developed by Rowe [6]. Independently and using different techniques the same approach has been developed in plasma physics where it is called cluster Hartree-Fock theory [7]. Much earlier, quite similar ideas were also put forward by the Japanese school of Marumori et al. [8]. The recent theoretical developments along this line and the various successes in applications to model cases [9-16] make us f rmly believe that there exists a great potentiality in this approach which is very general and can be easily adapted to practically any kind of many body problem where two body correlations or even higher correlations are of importance.

The SCRPA scheme has indeed, on the level of the two body correlations or f uctuations characterized by a correlated pair of Fermion operators like $Q_{v}^{\dagger}=\sum_{k k^{\prime}} X_{k k^{\prime}}^{v} a_{k}^{\dagger} a_{k^{\prime}}^{\dagger}$, or $\sum_{k k^{\prime}} X_{k k^{\prime}}^{v} a_{k}^{\dagger} a_{k^{\prime}}$, some aspects quite analogous to ordinary Hartree-Fock theory characterized by the single operator $q_{\alpha}^{\dagger}=\sum_{k} \varphi_{k}^{\alpha} a_{k}^{\dagger}$. As we will show in the main text, standard Hartree-Fock theory and SCRPA or Cluster Hartree-Fock theory can be derived from a common variational principle leading to the equations.

$$
\begin{aligned}
\left\langle\left\{\delta_{q},\left[H, q_{\alpha}^{\dagger}\right]\right\}\right\rangle & =\varepsilon_{\alpha}\left\langle\left\{\delta_{q}, q_{\alpha}^{\dagger}\right\}\right\rangle, \\
\left\langle\left[\delta Q,\left[H, Q_{v}^{\dagger}\right]\right]\right\rangle & =E_{v}\left\langle\left[\delta Q, Q_{v}^{\dagger}\right]\right\rangle,
\end{aligned}
$$

where $\{$,$\} is the anticommutator and [,] the commutator, and \langle\cdots\rangle$ stands for the mean value with respect to vacua def ned by $q_{\alpha}|0\rangle=0$ or $Q_{\nu}|0\rangle=0$. Equations (1) and (2) are not only formally very similar. Also their physical content is quite analogous. Whereas (1) describes the motion of one particle in the average f eld created by all the others, the second Eq. (2) describes the motion of a quantal $f$ uctuation (a correlated Fermion pair) in the average $f$ eld created by all the other $f$ uctuations (pairs). A nucleus, for example, can be considered in some approximation as a gas of independent zero point vibrations (elementary excitations) as well as a gas of independent particles. Both gases create their own mean f eld and in general they will be coupled.

As with any theory for correlation functions also SCRPA is very diff cult and demanding with respect to numerical implementation in the general case. However, the recent solution of the many level pairing model is suff ciently complex to state that with respect to a brute force diagonalization, the SCRPA gives a tremendous simplif cation of the solution of the many body problem with very good accuracy which, at least for the case considered, does not fail in any qualitative respects. It is our intention in this work to take up again the SCRPA solution of the many level pairing model of Ref. [15] in more detail. In the f rst place we circumvent an approximation used in [15] so that in this work the SCRPA scheme is carried through in full without any approximation. On the basis of this achievement we will then be able to discuss the advantages and eventual shortcomings of the method. We also will be able to assess the def ciency of the approximation we adopted in [15] as well as other more severe simplifying reductions of the full SCRPA scheme such as the renormalized RPA (r-RPA) which, because of its relatively simple numerical structure, has become quite popular in the recent past [17-19].

In detail the paper is organized as follows: in Section 2 the picket-fence model is introduced, in Section 3 it is reformulated to make explicit its particle-hole symmetry, in Section 4 the SCRPA formalism is presented, in Section 5 the occupation numbers and correlation functions needed to close the equations are constructed, in Section 6 the approximations used to obtain simpler RPA formalisms are discussed, and in Section 7 sum rules related with the conservation of the particle number are presented. The explicit construction and the problems associated with the SCRPA ground state are discussed in Section 8, numerical results are shown in Section 9, and the conclusions in Section 10. Some useful mathematical results are given in the Appendix. 


\section{THE PICKET-FENCE MODEL}

The picket fence or multilevel pairing model has been introduced by Richardson in 1966 [20] to describe deformed nuclei. The model has the great advantage of being non-trivial (in the sense that it cannot straightforwardly be diagonalised for an arbitrary number of levels) and still being exactly solvable (by some kind of Bethe ansatz). However, in nuclear physics, aside from some rare considerations [21], the model has not been exploited very much probably because it has been judged too crude for the description of real nuclei. Still the model contains very interesting physics and it has recently been revived in the context of ultra-small superconducting metallic grains [22]. One of the most interesting aspects of the model is that the exact solution reveals a transition between the superf uid (or superconducting) regime and the normal state which is completely smooth, i.e., no sign of any abrupt phase transition from one state to the other can be detected as a function of the system parameters [15].

The original Hamiltonian of the model is given by

$$
H=\sum_{i=1}^{\Omega}\left(\varepsilon_{i}-\lambda\right) N_{i}-G \sum_{i, j=1}^{\Omega} P_{i}^{\dagger} P_{j},
$$

with

$$
N_{i}=c_{i}^{\dagger} c_{i}+c_{-i}^{\dagger} c_{-i}, \quad P_{i}^{\dagger}=c_{i}^{\dagger} c_{-i}^{\dagger},
$$

where $c_{i}^{\dagger}$ creates a particle in the $i$ th level with $j=\frac{1}{2}$ and $m=\frac{1}{2}$ and $c_{-i}^{\dagger}$ with $m=-\frac{1}{2}$. $\Omega$ is the total number of levels, $G$ is the pairing interaction strength, and $\varepsilon_{i}=i \varepsilon$. The chemical potential $\lambda$ will be def ned such that the Hamiltonian preserves particle-hole symmetry. Each level has degeneracy two and we will assume that the system is half flled with number of pairs $N=\Omega / 2$.

The particle $(p)$ and hole $(h)$ states are def ned by

$$
N_{h}|H F\rangle=2, \quad N_{p}|H F\rangle=0,
$$

where $|H F\rangle$ is the ground state of the Hamiltonian (3) with $\mathrm{G}=0$. The particle states $p$ correspond to $\varepsilon_{p}>\lambda$ and the holes $h$ to $\varepsilon_{p}<\lambda$.

In this case, with no partial occupations allowed, the following relation is fulf lled,

$$
P_{i}^{\dagger} P_{i}+P_{i} P_{i}^{\dagger}=1
$$

which implies

$$
N_{i}=2 P_{i}^{\dagger} P_{i}
$$

\section{THE PARTICLE-HOLE SYMMETRY}

The commutation relations between the operators def ned in (2) are

$$
\left[P_{i}, P_{j}^{\dagger}\right]=\delta_{i j}\left(1-N_{i}\right), \quad\left[N_{i}, P_{J}^{\dagger}\right]=2 \delta_{i j} P_{j}^{\dagger}, \quad\left[N_{i}, P_{j}\right]=-2 \delta_{i j} P_{j}
$$


To make explicit the particle-hole symmetry we will make the following particle-hole conjugation

$$
c_{p}=d_{p}, \quad c_{\bar{p}}=d_{\bar{p}}, \quad c_{h}=d_{\bar{h}}^{\dagger}, \quad c_{\bar{h}}^{-}=-d_{h}^{\dagger} .
$$

The new operators $M, Q$, and $Q^{\dagger}$ in terms of $d^{\dagger}(d)$ are

$$
N_{h}=2-M_{h}, \quad N_{p}=M_{p}, \quad P_{h}^{\dagger}=-Q_{h}, \quad P_{p}^{\dagger}=Q_{p}^{\dagger} .
$$

Their commutation relations are

$$
\begin{array}{lll}
{\left[Q_{p}, Q_{p^{\prime}}^{\dagger}\right]=\delta_{p p^{\prime}}\left(1-M_{p}\right),} & {\left[M_{p}, Q_{p^{\prime}}^{\dagger}\right]=2 \delta_{p p^{\prime}} Q_{p}^{\dagger},} & {\left[M_{p}, Q_{p^{\prime}}\right]=-2 \delta_{p p^{\prime}} Q_{p},} \\
{\left[Q_{h}, Q_{h^{\prime}}^{\dagger}\right]=\delta_{h h^{\prime}}\left(1-M_{h}\right),} & {\left[M_{h}, Q_{h^{\prime}}^{\dagger}\right]=2 \delta_{h h^{\prime}} Q_{h}^{\dagger},} & {\left[M_{h}, Q_{h^{\prime}}\right]=-2 \delta_{h h^{\prime}} Q_{h} .}
\end{array}
$$

The single particle energies are $\varepsilon_{p}=\varepsilon(N+p)$ and $\varepsilon_{h}=\varepsilon(N-h+1)$, with $p, h=1, \ldots, N$, and $N=\frac{1}{2} \Omega$. Particles (p) and holes (h) are numbered starting from the level closest to the Fermi level. We use the chemical potential to restore the particle-hole symmetry:

$$
\lambda=\varepsilon\left(N+\frac{1}{2}\right)-\frac{G}{2} .
$$

With this def nition the Hamiltonian reduces to

$$
\begin{aligned}
H= & -\varepsilon N^{2}+\sum_{p=h=1}^{N}\left[\varepsilon\left(p-\frac{1}{2}\right)+\frac{G}{2}\right]\left(M_{p}+M_{h}\right) \\
& -G \sum_{p p^{\prime}} Q_{p}^{\dagger} Q_{p^{\prime}}-G \sum_{h h^{\prime}} Q_{h}^{\dagger} Q_{h^{\prime}}+G \sum_{p h}\left(Q_{p}^{\dagger} Q_{h}^{\dagger}+Q_{p} Q_{h}\right) .
\end{aligned}
$$

In this form the complete symmetry between particle and hole states becomes evident. This will greatly facilitate later the formal and numerical aspects of our theory.

\section{THE SCRPA FORMALISM}

The basic ingredients of the SCRPA approach in the particle-particle channel are the two particle addition operator

$$
A_{\mu}^{\dagger}=\sum_{p} X_{p}^{\mu} \bar{Q}_{p}^{\dagger}-\sum_{h} Y_{h}^{\mu} \bar{Q}_{h},
$$

and the removal operator

$$
R_{\lambda}^{\dagger}=-\sum_{p} Y_{p}^{\lambda} \bar{Q}_{p}+\sum_{h} X_{h}^{\lambda} \bar{Q}_{h}^{\dagger},
$$

where $\bar{Q}_{p}=Q_{p} / \sqrt{1-\left\langle M_{p}\right\rangle}$ and $\bar{Q}_{h}=Q_{h} / \sqrt{1-\left\langle M_{h}\right\rangle}$. 
Following Baranger in his derivation for the single particle mean f eld Eq. [23], we def ne the following mean excitation energy

$$
\begin{aligned}
\Omega_{\mu}= & \left\{\sum_{\alpha(N+2)}\left(E_{N+2}^{\alpha}-E_{N+2}^{0}\right)\left|\left\langle\alpha\left|A_{\mu}^{\dagger}\right| 0\right\rangle\right|^{2}+\sum_{\beta(N-2)}\left(E_{N-2}^{\beta}-E_{N-2}^{0}\right)\right. \\
& \left.\left.\times\left|\left\langle\beta\left|A_{\mu}\right| 0\right\rangle\right|^{2}+2 \mu^{(+)} \sum_{\alpha}\left|\left\langle\alpha\left|A_{\mu}^{\dagger}\right| 0\right\rangle\right|^{2}-2 \mu^{(-)} \sum_{\beta}\left|\left\langle\beta\left|A_{\mu}\right| 0\right\rangle\right|^{2}\right]\right\} \\
& \left\{\sum_{\alpha(N+2)}\left|\left\langle\alpha\left|A_{\mu}^{\dagger}\right| 0\right\rangle\right|^{2}-\sum_{\beta(N-2)}\left|\left\langle\beta\left|A_{\mu}\right| 0\right\rangle\right|^{2}\right\}
\end{aligned}
$$

where $2 \mu^{( \pm)}=( \pm) 1 / 2\left(E_{N+/-2}^{0}-E_{N}^{0}\right)$ are the chemical potentials and $E_{N}^{\alpha, \beta}$ are the in principle exact eigenvalues of $H$ of Eq. (1) and $|\alpha\rangle,|\beta\rangle,|0\rangle$ are the corresponding exact eigenstates.

Expression (16) can be considered as an average excitation energy taking into account the spectra of both the $N+2$ and $N-2$ systems. In fact $\Omega_{\mu}$ represents the energy weighted sum rule over both spectra divided by the non-energy weighted sum rule.

Indeed expression (16) can also be written as

$$
\Omega_{\mu}=\frac{\left\langle 0\left|\left[A_{\mu},\left[H, A_{\mu}^{\dagger}\right]\right]\right| 0\right\rangle}{\left\langle 0\left|\left[A_{\mu}, A_{\mu}^{\dagger}\right]\right| 0\right\rangle}
$$

which makes the analogy with the usual energy weighted sum rule in the particle-hole channel quite obvious. The terms in (16) involving the chemical potentials $\mu^{( \pm)}$are needed in order to give the correct origin of the energy spectra. This is best seen in supposing that $\mu^{(+)}=\mu^{(-)}$which leads to

$$
\Omega_{\mu}-2 \mu=\frac{\sum_{\alpha}\left(E_{N+2}^{\alpha}-E_{N+2}^{0}\right)\left|\left\langle\alpha\left|A_{\mu}^{\dagger}\right| 0\right\rangle\right|^{2}+\sum_{\beta}\left(E_{N-2}^{\beta}-E_{N-2}^{0}\right)\left|\left\langle\beta\left|A_{\mu}\right| 0\right\rangle\right|^{2}}{\left\langle 0\left|\left[A_{\mu}, A_{\mu}^{\dagger}\right]\right| 0\right\rangle}
$$

It means that the energy weighting starts from two times the chemical potential, as it should be.

The $\mathrm{f}$ rst step is now to minimize the average two particle energy (16) with respect to the amplitudes $X, Y$. This leads straightforwardly to the following set of equations,

$$
\left(\begin{array}{cc}
A & B \\
-B & C
\end{array}\right)\left(\begin{array}{l}
X \\
Y
\end{array}\right)=E\left(\begin{array}{l}
X \\
Y
\end{array}\right)
$$

where

$$
\begin{aligned}
A_{p p^{\prime}}= & \left\langle 0\left|\left[\bar{Q}_{p},\left[H, \bar{Q}_{p^{\prime}}^{\dagger}\right]\right]\right| 0\right\rangle \\
= & \delta_{p p^{\prime}}\left\{2\left(\epsilon\left(p-\frac{1}{2}\right)+\frac{G}{2}\right)+2 \frac{G}{1-\left\langle M_{p}\right\rangle}\left\langle\left(\sum_{p_{1}} Q_{p_{1}}^{\dagger}-\sum_{h_{1}} Q_{h_{1}}\right) Q_{p}\right\rangle\right\} \\
& -G \frac{\left\langle\left(1-M_{p}\right)\left(1-M_{p^{\prime}}\right)\right\rangle}{\sqrt{\left(1-\left\langle M_{p}\right\rangle\right)\left(1-\left\langle M_{p^{\prime}}\right\rangle\right)}},
\end{aligned}
$$




$$
\begin{aligned}
B_{p h}= & \left\langle 0\left|\left[\bar{Q}_{p},\left[H, \bar{Q}_{h}^{\dagger}\right]\right]\right| 0\right\rangle=G \frac{\left\langle\left(1-M_{p}\right)\left(1-M_{h}\right)\right\rangle}{\sqrt{\left(1-\left\langle M_{p}\right\rangle\right)\left(1-\left\langle M_{h}\right\rangle\right)}}, \\
C_{h h^{\prime}}= & \left\langle 0\left|\left[\bar{Q}_{h},\left[H, \bar{Q}_{h^{\prime}}^{\dagger}\right]\right]\right| 0\right\rangle \\
= & \delta_{h h^{\prime}}\left\{-2\left(\epsilon\left(h-\frac{1}{2}\right)+\frac{G}{2}\right)+2 \frac{G}{1-\left\langle M_{h}\right\rangle}\left\langle Q_{h}^{\dagger}\left(-\sum_{p_{1}} Q_{p_{1}}^{\dagger}+\sum_{h_{1}} Q_{h_{1}}\right)\right\rangle\right\} \\
& +G \frac{\left\langle\left(1-M_{h}\right)\left(1-M_{h^{\prime}}\right)\right\rangle}{\sqrt{\left(1-\left\langle M_{h}\right\rangle\right)\left(1-\left\langle M_{h^{\prime}}\right\rangle\right)}} .
\end{aligned}
$$

Due to the particle-hole symmetry, the removal mode satisf es exactly the same equations, implying that both modes have exactly the same excitation energies and wave functions. To advance this conclusion we are assuming the following relations,

$$
\begin{aligned}
\left\langle M_{p}\right\rangle & =\left\langle M_{h=p}\right\rangle, & & \\
\left\langle Q_{p}^{\dagger} Q_{p^{\prime}}\right\rangle & =\left\langle Q_{h=p}^{\dagger} Q_{h^{\prime}=p^{\prime}}\right\rangle, & & \left\langle Q_{h} Q_{p}\right\rangle=\left\langle Q_{p}^{\dagger} Q_{h}^{\dagger}\right\rangle \\
\left\langle M_{p} M_{p^{\prime}}\right\rangle & =\left\langle M_{h=p} M_{h^{\prime}=p^{\prime}}\right\rangle, & & \left\langle M_{h} M_{p}\right\rangle=\left\langle M_{h^{\prime}=p} M_{p^{\prime}=h}\right\rangle,
\end{aligned}
$$

which are shown below to be consistent with the above equations.

It means that

$$
X_{p}^{\mu}= \pm X_{h=p}^{\lambda=\mu}, \quad Y_{h}^{\mu}= \pm Y_{p=h}^{\lambda=\mu}
$$

The forward and backward amplitudes $X, Y$ fulf 11 the normalization conditions

$$
\begin{aligned}
\sum_{p} X_{p}^{\mu} X_{p}^{\mu^{\prime}}-\sum_{h} Y_{h}^{\mu} Y_{h}^{\mu^{\prime}} & =\delta_{\mu \mu^{\prime}} \\
\sum_{h} X_{h}^{\lambda} X_{h}^{\lambda^{\prime}}-\sum_{p} Y_{p}^{\lambda} Y_{p}^{\lambda^{\prime}} & =\delta_{\lambda \lambda^{\prime}} \\
\sum_{p} X_{p}^{\mu} Y_{p}^{\lambda}-\sum_{h} X_{h}^{\lambda} Y_{h}^{\mu} & =0
\end{aligned}
$$

and the closure relations

$$
\begin{aligned}
& \sum_{\mu} X_{p}^{\mu} X_{p^{\prime}}^{\mu}-\sum_{\lambda} Y_{p}^{\lambda} Y_{p^{\prime}}^{\lambda}=\delta_{p p^{\prime}}, \\
& \sum_{\lambda} X_{h}^{\lambda} X_{h^{\prime}}^{\lambda}-\sum_{\mu} Y_{h}^{\mu} Y_{h^{\prime}}^{\mu}=\delta_{h h^{\prime}}, \\
& \sum_{\lambda} X_{h}^{\lambda} Y_{p}^{\lambda}-\sum_{\mu} X_{p}^{\mu} Y_{h}^{\mu}=0 .
\end{aligned}
$$

The expectation values of the commutators are

$$
\begin{gathered}
\left\langle\left[R_{\lambda}, R_{\lambda^{\prime}}^{\dagger}\right]\right\rangle=\delta_{\lambda \lambda^{\prime}}, \quad\left\langle\left[A_{\mu}, A_{\mu^{\prime}}^{\dagger}\right]\right\rangle=\delta_{\mu \mu^{\prime}}, \\
\left\langle\left[A_{\mu}^{\dagger}, R_{\lambda^{\prime}}^{\dagger}\right]\right\rangle=\left\langle\left[A_{\mu}, R_{\lambda^{\prime}}\right]\right\rangle=0, \quad\left[R_{\lambda}, A_{\mu}^{\dagger}\right]=0 .
\end{gathered}
$$


With the help of these equations we can now invert (14), (15) which yields

$$
\begin{aligned}
Q_{p}^{\dagger} & =\sqrt{1-\left\langle M_{p}\right\rangle}\left[\sum_{\mu} X_{p}^{\mu} A_{\mu}^{\dagger}+\sum_{\lambda} Y_{p}^{\lambda} R_{\lambda}\right], \\
Q_{h} & =\sqrt{1-\left\langle M_{h}\right\rangle}\left[\sum_{\lambda} X_{h}^{\lambda} R^{\lambda}+\sum_{\mu} Y_{h}^{\mu} A_{\mu}^{\dagger}\right] .
\end{aligned}
$$

An important point to recognize at this step is the fact that the above SCRPA equations can also be derived using the equation of motion method advocated, for example, by Rowe [6]. A basic ingredient to this latter method is that one assumes the existence of a vacuum state $|0\rangle \equiv|S C R P A\rangle$ such that

$$
A_{\mu}|S C R P A\rangle=R_{\lambda}|S C R P A\rangle=0 .
$$

Therefore Eq. (28) must be considered as an inherent additional relation belonging to the whole SCRPA scheme. The expectation values in (20) shall then be evaluated with this ground state and, as we will show in a moment, this will permit us to entirely close the system of equations.

\section{CLOSING THE SYSTEM OF EQUATIONS}

The aim is now to express the expectation values which f gure in the RPA-matrix (20) entirely and without approximation by the amplitudes $X, Y$. Using $(27),(28)$ this can easily be achieved for most of the expectation values. Indeed one directly verif es

$$
\begin{aligned}
\left\langle Q_{p}^{\dagger} Q_{p^{\prime}}\right\rangle & =\sqrt{\left(1-\left\langle M_{p}\right\rangle\right)\left(1-\left\langle M_{p^{\prime}}\right\rangle\right)} \sum_{\lambda} Y_{p}^{\lambda} Y_{p^{\prime}}^{\lambda}, \\
\left\langle Q_{h}^{\dagger} Q_{h^{\prime}}\right\rangle & =\sqrt{\left(1-\left\langle M_{h}\right\rangle\right)\left(1-\left\langle M_{h^{\prime}}\right\rangle\right)} \sum_{\mu} Y_{h}^{\mu} Y_{h^{\prime}}^{\mu}, \\
\left\langle Q_{h} Q_{p}\right\rangle & =\left\langle Q_{p}^{\dagger} Q_{h}^{\dagger}\right\rangle=\sqrt{\left(1-\left\langle M_{h}\right\rangle\right)\left(1-\left\langle M_{p}\right\rangle\right)} \sum_{\lambda} Y_{p}^{\lambda} X_{h}^{\lambda} .
\end{aligned}
$$

Using $(7,10)$ it is direct to show that

$$
\left\langle M_{p}\right\rangle=1-\frac{1}{1+2 \sum_{\lambda}\left(Y_{p}^{\lambda}\right)^{2}}, \quad\left\langle M_{h}\right\rangle=1-\frac{1}{1+2 \sum_{\mu}\left(Y_{h}^{\mu}\right)^{2}},
$$

which together with (22) implies

$$
\left\langle M_{p=i}\right\rangle=\left\langle M_{h=i}\right\rangle,
$$

ref ecting again the particle-hole symmetry. Using (22) and (31) it is also direct to show that

$$
\left\langle Q_{p}^{\dagger} Q_{p^{\prime}}\right\rangle=\left\langle Q_{h=p}^{\dagger} Q_{h^{\prime}=p^{\prime}}\right\rangle
$$

These are the frst three of the f ve equalities advanced in (21). 
Knowing these expectation values we can evaluate the SCRPA ground state energy:

$$
\begin{aligned}
\langle H\rangle= & -\varepsilon N^{2}+\sum_{p=h=1}^{N}\left[\varepsilon\left(p-\frac{1}{2}\right)+\frac{G}{2}\right]\left(\left\langle M_{p}\right\rangle+\left\langle M_{h}\right\rangle\right) \\
& -G \sum_{p p^{\prime}}\left\langle Q_{p}^{\dagger} Q_{p^{\prime}}\right\rangle-G \sum_{h h^{\prime}}\left\langle Q_{h}^{\dagger} Q_{h^{\prime}}\right\rangle+G \sum_{p h}\left(\left\langle Q_{p}^{\dagger} Q_{h}^{\dagger}+Q_{p} Q_{h}\right\rangle\right) .
\end{aligned}
$$

The SCRPA correlation energy is

$$
E_{\text {corr }}^{S C R P A}=\langle H\rangle+\varepsilon N^{2} .
$$

For comparison, the RPA correlation energy is

$$
E_{\text {corr }}^{R P A}=-\sum_{\mu} E_{\mu} \sum_{p}\left|Y_{p}^{\mu}\right|^{2} .
$$

In order to fully close the set of SCRPA equations we still must express the correlation functions $\left\langle M_{i} M_{j}\right\rangle$ through the RPA amplitudes. This can also be done exactly, though it is somewhat involved. For this reason we approximated $\left\langle M_{i} M_{j}\right\rangle$ by $\left\langle M_{i}\right\rangle\left\langle M_{j}\right\rangle$ in [15]. In what follows we now present the full derivation.

Using (4) and (7) it is direct to demonstrate that

$$
N_{i} N_{i}=2 N_{i},
$$

which implies that

$$
M_{p} M_{p}=2 M_{p}, \quad M_{h} M_{h}=2 M_{h} .
$$

It is also simple to demonstrate that

$$
N_{i} N_{j}=4 P_{i}^{\dagger} P_{j}^{\dagger} P_{j} P_{i} \quad \text { for } i \neq j,
$$

which implies the three sets of equations

$$
\begin{aligned}
& M_{p} M_{p^{\prime}}=4 Q_{p}^{\dagger} Q_{p^{\prime}}^{\dagger} Q_{p^{\prime}} Q_{p} \quad \text { for } p \neq p^{\prime}, \\
& M_{p} M_{h}=M_{p}+M_{h}-2 Q_{p}^{\dagger} Q_{h} Q_{h}^{\dagger} Q_{p}-2 Q_{h}^{\dagger} Q_{p} Q_{p}^{\dagger} Q_{h}, \\
& M_{h} M_{h^{\prime}}=4 Q_{h}^{\dagger} Q_{h^{\prime}}^{\dagger} Q_{h^{\prime}} Q_{h} \quad \text { for } h \neq h^{\prime} .
\end{aligned}
$$

We have then $\Omega$ expectation values which are known:

$$
\left\langle M_{p} M_{p}\right\rangle=2\left\langle M_{p}\right\rangle, \quad\left\langle M_{h} M_{h}\right\rangle=2\left\langle M_{h}\right\rangle .
$$

For $p \neq p^{\prime}, h \neq h^{\prime}$ the equations are

$$
\begin{aligned}
\left\langle M_{p} M_{p^{\prime}}\right\rangle= & 4\left(1-\left\langle M_{p}\right\rangle\right)\left(1-\left\langle M_{p^{\prime}}\right\rangle\right) \sum_{\lambda \lambda^{\prime}} \sum_{\lambda_{1} \lambda_{2}} Y_{p}^{\lambda} Y_{p}^{\lambda^{\prime}} Y_{p^{\prime}}^{\lambda_{1}} Y_{p^{\prime}}^{\lambda_{2}}\left\langle R_{\lambda} R_{\lambda_{1}} R_{\lambda_{2}}^{\dagger} R_{\lambda^{\prime}}^{\dagger}\right\rangle \\
\left\langle M_{p} M_{h}\right\rangle= & \left\langle M_{p}\right\rangle+\left\langle M_{h}\right\rangle-2\left(1-\left\langle M_{p}\right\rangle\right)\left(1-\left\langle M_{h}\right\rangle\right) \sum_{\lambda \lambda^{\prime}} \sum_{\lambda_{1} \lambda_{2}} Y_{p}^{\lambda} Y_{p}^{\lambda^{\prime}} X_{h}^{\lambda_{1}} X_{h}^{\lambda_{2}}\left\langle R_{\lambda} R_{\lambda_{1}} R_{\lambda_{2}}^{\dagger} R_{\lambda^{\prime}}^{\dagger}\right\rangle \\
& -2\left(1-\left\langle M_{p}\right\rangle\right)\left(1-\left\langle M_{h}\right\rangle\right) \sum_{\mu \mu^{\prime}} \sum_{\mu_{1} \mu_{2}} Y_{h}^{\mu} Y_{h}^{\mu^{\prime}} X_{p}^{\mu_{1}} X_{p}^{\mu_{2}}\left\langle A_{\mu} A_{\mu_{1}} A_{\mu_{2}}^{\dagger} A_{\mu^{\prime}}^{\dagger}\right\rangle, \\
\left\langle M_{h} M_{h^{\prime}}\right\rangle= & 4\left(1-\left\langle M_{h}\right\rangle\right)\left(1-\left\langle M_{h^{\prime}}\right\rangle\right) \sum_{\mu \mu^{\prime}} \sum_{\mu_{1} \mu_{2}} Y_{h}^{\mu} Y_{h}^{\mu^{\prime}} Y_{h^{\prime}}^{\mu_{1}} Y_{h^{\prime}}^{\mu_{2}}\left\langle A_{\mu} A_{\mu_{1}} A_{\mu_{2}}^{\dagger} A_{\mu^{\prime}}^{\dagger}\right\rangle .
\end{aligned}
$$


Using the identities given in the Appendix we build up the system of equations

$$
\begin{aligned}
& \sum_{h_{1} h_{2}}\left\langle M_{h_{1}} M_{h_{2}}\right\rangle\left(B_{h_{1} h_{2}}^{h h^{\prime}}-\frac{\delta_{h h_{1}} \delta_{h^{\prime} h_{2}}}{4\left(1-\left\langle M_{h_{1}}\right\rangle\right)^{2}\left(1-\left\langle M_{h_{2}}\right\rangle\right)^{2}}\right) \\
& \quad+\sum_{h_{1} p_{1}}\left\langle M_{h_{1}} M_{p_{1}}\right\rangle C_{h_{1} p_{1}}^{h h^{\prime}}+\sum_{p_{1} p_{2}}\left\langle M_{p_{1}} M_{p_{2}}\right\rangle D_{p_{1} p_{2}}^{h h^{\prime}}=F_{h h^{\prime}} \\
& \sum_{h_{1} h_{2}}\left\langle M_{h_{1}} M_{h_{2}}\right\rangle B_{h_{1} h_{2}}^{h p}+\sum_{h_{1} p_{1}}\left\langle M_{h_{1}} M_{p_{1}}\right\rangle\left(C_{h_{1} p_{1}}^{h p}+\frac{\delta_{h h_{1}} \delta_{p p_{1}}}{2\left(1-\left\langle M_{h_{1}}\right\rangle\right)^{2}\left(1-\left\langle M_{p_{1}}\right\rangle\right)^{2}}\right) \\
& \quad+\sum_{p_{1} p_{2}}\left\langle M_{p_{1}} M_{p_{2}}\right\rangle D_{p_{1} p_{2}}^{h p}=F_{h p}, \\
& \sum_{h_{1} h_{2}}\left\langle M_{h_{1}} M_{h_{2}}\right\rangle B_{h_{1} h_{2}}^{p p^{\prime}}+\sum_{h_{1} p_{1}}\left\langle M_{h_{1}} M_{p_{1}}\right\rangle C_{h_{1} p_{1}}^{p p^{\prime}}+\sum_{p_{1} p_{2}}\left\langle M_{p_{1}} M_{p_{2}}\right\rangle \\
& \quad \times\left(D_{p_{1} p_{2}}^{p p^{\prime}}-\frac{\delta_{p p_{1}} \delta_{p^{\prime} p_{2}}}{4\left(1-\left\langle M_{p_{1}}\right\rangle\right)^{2}\left(1-\left\langle M_{p_{2}}\right\rangle\right)^{2}}\right)=F_{p p^{\prime}},
\end{aligned}
$$

where we have introduced the auxiliary matrices

$$
\begin{aligned}
& F_{p p^{\prime}}=-2 \sum_{p_{1}}\left(1-\left\langle M_{p_{1}}\right\rangle\right) \mathcal{Y}_{p p_{1}}^{2} \mathcal{Y}_{p^{\prime} p_{1}}^{2}+2 \sum_{h_{1}}\left(1-\left\langle M_{h_{1}}\right\rangle\right) \mathcal{Z}_{p h_{1}}^{2} \mathcal{Z}_{p^{\prime} h_{1}}^{2} \\
& -\sum_{h_{1} h_{2}} B_{h_{1} h_{2}}^{p p^{\prime}}\left(1-\left\langle M_{h_{1}}\right\rangle-\left\langle M_{h_{2}}\right\rangle\right)-\sum_{h_{1} p_{1}} C_{h_{1} p_{1}}^{p p^{\prime}}\left(1-\left\langle M_{p_{1}}\right\rangle-\left\langle M_{h_{1}}\right\rangle\right) \\
& -\sum_{p_{1} p_{2}} D_{p_{1} p_{2}}^{p p^{\prime}}\left(1-\left\langle M_{p_{1}}\right\rangle-\left\langle M_{p_{2}}\right\rangle\right) \\
& B_{h_{1} h_{2}}^{p p^{\prime}}=\left(\mathcal{Z}_{p h_{1}} \mathcal{Z}_{p^{\prime} h_{2}}\right)^{2}+\mathcal{Z}_{p h_{1}} \mathcal{Z}_{p^{\prime} h_{1}} \mathcal{Z}_{p h_{2}} \mathcal{Z}_{p^{\prime} h_{2}}, \\
& C_{h_{1} p_{1}}^{p p^{\prime}}=-\left(\mathcal{Y}_{p p_{1}} \mathcal{Z}_{p^{\prime} h_{1}}+\mathcal{Y}_{p^{\prime} p_{1}} \mathcal{Z}_{p h_{1}}\right)^{2} \text {, } \\
& D_{p_{1} p_{2}}^{p p^{\prime}}=\left(\mathcal{Y}_{p p_{1}} \mathcal{Y}_{p^{\prime} p_{2}}\right)^{2}+\mathcal{Y}_{p p_{1}} \mathcal{Y}_{p^{\prime} p_{2}} \mathcal{Y}_{p^{\prime} p_{1}} \mathcal{Y}_{p p_{2}} \\
& F_{h p}=\left(\left\langle M_{h}\right\rangle+\left\langle M_{p}\right\rangle\right) /\left(2\left(1-\left\langle M_{h}\right\rangle\right)^{2}\left(1-\left\langle M_{p}\right\rangle\right)^{2}\right)-2 \sum_{p_{1}}\left(1-\left\langle M_{p_{1}}\right\rangle\right)\left(\mathcal{Y}_{p p_{1}}^{2} \mathcal{Z}_{p_{1} h}^{2}-\mathcal{X}_{p p_{1}}^{2} \mathcal{Z}_{h p_{1}}^{2}\right) \\
& -2 \sum_{h_{1}}\left(1-\left\langle M_{h_{1}}\right\rangle\right)\left(\mathcal{Z}_{h_{1} p}^{2} \mathcal{Y}_{h h_{1}}^{2}-\mathcal{Z}_{p h_{1}}^{2} \mathcal{X}_{h h_{1}}^{2}\right)-\sum_{h_{1} h_{2}} B_{h_{1} h_{2}}^{p h}\left(1-\left\langle M_{h_{1}}\right\rangle-\left\langle M_{h_{2}}\right\rangle\right) \\
& -\sum_{h_{1} p_{1}} C_{h_{1} p_{1}}^{p h}\left(1-\left\langle M_{p_{1}}\right\rangle-\left\langle M_{h_{1}}\right\rangle\right)-\sum_{p_{1} p_{2}} D_{p_{1} p_{2}}^{p h}\left(1-\left\langle M_{p_{1}}\right\rangle-\left\langle M_{p_{2}}\right\rangle\right), \\
& B_{h_{1} h_{2}}^{h p}=\left(\mathcal{Y}_{h h_{1}} \mathcal{Z}_{h_{2} p}\right)^{2}+\mathcal{Y}_{h h_{1}} \mathcal{Z}_{h_{2} p} \mathcal{Z}_{h_{1} p} \mathcal{Y}_{h h_{2}}+\left(\mathcal{X}_{h h_{1}} \mathcal{Z}_{p h_{2}}\right)^{2}+\mathcal{X}_{h h_{1}} \mathcal{Z}_{p h_{2}} \mathcal{Z}_{p h_{1}} \mathcal{X}_{h h_{2}} \\
& C_{h_{1} p_{1}}^{h p}=-\left(\mathcal{Y}_{p p_{1}} \mathcal{X}_{h h_{1}}+\mathcal{Z}_{p_{1} h} \mathcal{Z}_{p_{1} h} \mathcal{Z}_{p h_{1}}\right)^{2}-\left(\mathcal{Y}_{h h_{1}} \mathcal{X}_{p p_{1}}+\mathcal{Z}_{h_{1} p} \mathcal{Z}_{h p_{1}}\right)^{2} \\
& D_{p_{1} p_{2}}^{h p}=\left(\mathcal{Z}_{p_{1} h} \mathcal{Y}_{p p_{2}}\right)^{2}+\mathcal{Z}_{p_{1} h} \mathcal{Y}_{p p_{1}} \mathcal{Z}_{p_{2} h} \mathcal{Y}_{p p_{2}}+\left(\mathcal{Z}_{h p_{1}} \mathcal{X}_{p p_{2}}\right)^{2}+\mathcal{Z}_{h p_{1}} \mathcal{X}_{p p_{1}} \mathcal{Z}_{h p_{2}} \mathcal{X}_{p p_{2}}
\end{aligned}
$$




$$
\begin{aligned}
F_{h h^{\prime}}= & 2 \sum_{p_{1}}\left(1-\left\langle M_{p_{1}}\right\rangle\right) \mathcal{Z}_{h p_{1}}^{2} \mathcal{Z}_{h^{\prime} p_{1}}^{2}-2 \sum_{h_{1}}\left(1-\left\langle M_{h_{1}}\right\rangle\right) \mathcal{Y}_{h^{\prime} h_{1}}^{2} \mathcal{Y}_{h h_{1}}^{2} \\
& -\sum_{h_{1} h_{2}} B_{h_{1} h_{2}}^{h^{\prime} h}\left(1-\left\langle M_{h_{1}}\right\rangle-\left\langle M_{h_{2}}\right\rangle\right)-\sum_{h_{1} p_{1}} C_{h_{1} p_{1}}^{h^{\prime} h}\left(1-\left\langle M_{p_{1}}\right\rangle-\left\langle M_{h_{1}}\right\rangle\right) \\
& -\sum_{p_{1} p_{2}} D_{p_{1} p_{2}}^{h^{\prime} h}\left(1-\left\langle M_{p_{1}}\right\rangle-\left\langle M_{p_{2}}\right\rangle\right), \\
B_{h_{1} h_{2}}^{h h^{\prime}}= & \left(\mathcal{Y}_{h^{\prime} h_{1}} \mathcal{Y}_{h h_{2}}\right)^{2}+\mathcal{Y}_{h^{\prime} h_{1}} \mathcal{Y}_{h h_{1}} \mathcal{Y}_{h^{\prime} h_{2}} \mathcal{Y}_{h h_{2}}, \\
C_{h_{1} p_{1}}^{h h^{\prime}}= & -\left(\mathcal{Z}_{h^{\prime} p_{1}} \mathcal{Y}_{h h_{1}}+\mathcal{Z}_{h p_{1}} \mathcal{Y}_{h^{\prime} h_{1}}\right)^{2}, \\
D_{p_{1} p_{2}}^{h h^{\prime}}= & \left(\mathcal{Z}_{h^{\prime} p_{1}} \mathcal{Z}_{h p_{2}}\right)^{2}+\mathcal{Z}_{h^{\prime} p_{1}} \mathcal{Z}_{h p_{2}} \mathcal{Z}_{h p_{1}} \mathcal{Z}_{h^{\prime} p_{2}} ;
\end{aligned}
$$

with

$$
\begin{array}{ll}
\mathcal{X}_{h h_{1}}=\sum_{\lambda} \frac{X_{h}^{\lambda} X_{h_{1}}^{\lambda}}{\sqrt{\left(1-\left\langle M_{h}\right\rangle\right)\left(1-\left\langle M_{h_{1}}\right\rangle\right)}}, & \mathcal{X}_{p p_{1}}=\sum_{\mu} \frac{X_{p}^{\mu} X_{p_{1}}^{\mu}}{\sqrt{\left(1-\left\langle M_{p}\right\rangle\right)\left(1-\left\langle M_{p_{1}}\right\rangle\right)}}, \\
\mathcal{Y}_{p p_{1}}=\sum_{\lambda} \frac{Y_{p}^{\lambda} Y_{p_{1}}^{\lambda}}{\sqrt{\left(1-\left\langle M_{p}\right\rangle\right)\left(1-\left\langle M_{p_{1}}\right\rangle\right)}}, & \mathcal{Y}_{h_{h_{1}}}=\sum_{\mu} \frac{Y_{h}^{\mu} Y_{h_{1}}^{\mu}}{\sqrt{\left(1-\left\langle M_{h}\right\rangle\right)\left(1-\left\langle M_{h_{1}}\right\rangle\right)}}, \\
Z_{p h}=\sum_{\lambda} \frac{Y_{p}^{\lambda} X_{h}^{\lambda}}{\sqrt{\left(1-\left\langle M_{h}\right\rangle\right)\left(1-\left\langle M_{P}\right\rangle\right)}}, & Z_{h p}=\sum_{\mu} \frac{Y_{h}^{\mu} X_{p}^{\mu}}{\sqrt{\left(1-\left\langle M_{h}\right\rangle\right)\left(1-\left\langle M_{p}\right\rangle\right)}} .
\end{array}
$$

Due to the symmetry $\left\langle M_{h} M_{p}\right\rangle=\left\langle M_{p} M_{h}\right\rangle$ there is no need to include an explicit equation for $\left\langle M_{p} M_{h}\right\rangle$.

Notice that the above matrices have the following symmetry properties

$$
\begin{array}{lll}
B_{h_{1} h_{2}}^{p p^{\prime}}=B_{h_{2} h_{1}}^{p^{\prime} p}, & C_{h_{1} p_{1}}^{p p^{\prime}}=C_{h_{1} p_{1}}^{p^{\prime} p}, & D_{p_{1} p_{2}}^{p p^{\prime}}=D_{p_{2} p_{1}}^{p^{\prime} p}, \\
B_{h_{1} h_{2}}^{h h^{\prime}}=B_{h_{2} h_{1}}^{h^{\prime} h}, & C_{h_{1} p_{1}}^{h h^{\prime}}=C_{h_{1} p_{1}}^{h^{\prime} h}, & D_{p_{1} p_{2}}^{h h^{\prime}}=D_{p_{2} p_{1}}^{h^{\prime} h} .
\end{array}
$$

They imply

$$
F_{p p^{\prime}}=F_{p^{\prime} p}, \quad F_{h h^{\prime}}=F_{h^{\prime} h},
$$

and guarantee that $\left\langle M_{p} M_{p^{\prime}}\right\rangle=\left\langle M_{p^{\prime}} M_{p}\right\rangle$ and $\left\langle M_{h} M_{h^{\prime}}\right\rangle=\left\langle M_{h^{\prime}} M_{h}\right\rangle$.

Due to the particle-hole symmetry, Eqs. (22) and (31), these matrices possess also the following symmetries

$$
\mathcal{X}_{h h^{\prime}}=\mathcal{X}_{p=h p^{\prime}=h^{\prime}}, \quad \mathcal{Y}_{p p^{\prime}}=\mathcal{Y}_{h=p h^{\prime}=p^{\prime}}, \quad Z_{p h}=Z_{h^{\prime}=p p^{\prime}=h},
$$

which ref ects in the $A, B, C, D$ matrices as

$$
\begin{array}{ll}
B_{h_{1} h_{2}}^{h h^{\prime}}=D_{p_{1}=h_{1} p_{2}=h_{2}}^{p=h p^{\prime}=h^{\prime}}, & D_{p_{1} p_{2}}^{h h^{\prime}}=B_{h_{1}=p_{1} h_{2}=p_{2}}^{p=h p^{\prime}=h^{\prime}}, \\
C_{h_{1} p_{1}}^{h h^{\prime}}=C_{h_{1} p_{1}}^{p=h p^{\prime}=h^{\prime}}, & F_{p p^{\prime}}=F_{h=p h^{\prime}=p^{\prime}},
\end{array}
$$


thus implying that the particle-hole symmetry is fulf lled in the following sense:

$$
\left\langle M_{p} M_{p^{\prime}}\right\rangle=\left\langle M_{h=p} M_{h^{\prime}=p^{\prime}}\right\rangle, \quad\left\langle M_{p} M_{h}\right\rangle=\left\langle M_{p^{\prime}=h} M_{h^{\prime}=p}\right\rangle .
$$

These are the last two equalities which were assumed valid in (21). They show that the whole formalism is consistent with the particle-hole symmetry. With these symmetry relations Eqs. (42), (43), (44) together with Eqs. (45)-(50) can be solved numerically.

\section{SIMPLER RPA FORMALISMS}

In a previous work the SCRPA equations (20) were solved under the approximation

$$
\left\langle M_{i} M_{j}\right\rangle \approx\left\langle M_{i}\right\rangle\left\langle M_{j}\right\rangle
$$

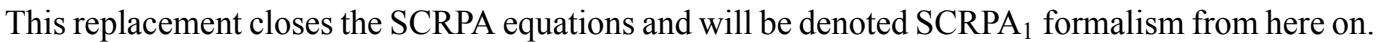

The renormalized RPA (r-RPA) formalism allows the consideration of the Pauli principle in a simpler way. The commutation relations between the operator $Q_{i}, Q_{j}^{\dagger}$ are replaced by their expectation value, i.e.,

$$
\left[Q_{i}, Q_{j}^{\dagger}\right] \rightarrow \delta_{i j}\left(1-\left\langle M_{i}\right\rangle\right)
$$

The RPA equations take the much simpler form

$$
\begin{aligned}
& A_{p p^{\prime}}^{\mathrm{r}-\mathrm{RPA}}=2 \delta_{p p^{\prime}}\left(\epsilon\left(p-\frac{1}{2}\right)+\frac{G}{2}\right)-G \sqrt{\left(1-\left\langle M_{p}\right\rangle\right)\left(1-\left\langle M_{p^{\prime}}\right\rangle\right)} \\
& B_{p h}^{\mathrm{r}-\mathrm{RPA}}=G \sqrt{\left(1-\left\langle M_{p}\right\rangle\right)\left(1-\left\langle M_{h}\right\rangle\right)} \\
& C_{h h^{\prime}}^{\mathrm{r}-\mathrm{RPA}}=-2 \delta_{h h^{\prime}}\left(\epsilon\left(h-\frac{1}{2}\right)+\frac{G}{2}\right)+G \sqrt{\left(1-\left\langle M_{h}\right\rangle\right)\left(1-\left\langle M_{h^{\prime}}\right\rangle\right)}
\end{aligned}
$$

They can be seen as a simplif cation of the $\mathrm{SCRPA}_{1}$ equations when the limit

$$
\left\langle Q_{i}^{\dagger} Q_{j}\right\rangle \rightarrow 0, \quad\left\langle Q_{i} Q_{j}\right\rangle \rightarrow 0
$$

is assumed valid. At the same time, we see that the correlation energy can only be obtained using the expression for $E_{c o r r}^{R P A}$.

The standard RPA equations [6] are usually built starting from the operators

$$
A_{0 \mu}^{\dagger}=\sum_{p} X_{p}^{0 \mu} Q_{p}^{\dagger}-\sum_{h} Y_{h}^{0 \mu} Q_{h}, \quad R_{0 \lambda}^{\dagger}=-\sum_{p} Y_{p}^{0 \lambda} Q_{p}+\sum_{h} X_{h}^{0 \lambda} Q_{h}^{\dagger} .
$$

They are def ned in terms of $Q_{i}, Q_{i}^{\dagger}$, in contrast with the SCRPA phonons which involve $\bar{Q}_{i}, \bar{Q}_{i}^{\dagger}$. The same changes are ref ected in the building of the RPA matrices $A, B, C$ in Eqs. (19), (20). In order to close the equations the so-called quasiboson approximation is postulated, which implies the replacement of the RPA vacuum $|0\rangle$ by the uncorrelated vacuum $|H F\rangle$ in the expectation values, in the form

$$
\langle 0|\left[A_{0 \mu}, A_{0 \mu^{\prime}}^{\dagger}|0\rangle \approx\langle H F|\left[A_{0 \mu}, A_{0 \mu^{\prime}}^{\dagger}|H F\rangle=\delta_{\mu, \mu^{\prime}} .\right.\right.
$$


In this way the standard RPA matrices for the picket fence model

$$
\begin{aligned}
& A_{p p^{\prime}}^{R P A}=2 \delta_{p p^{\prime}}\left(\epsilon\left(p-\frac{1}{2}\right)+\frac{G}{2}\right)-G, \\
& B_{p h}^{R P A}=G, \\
& C_{h h^{\prime}}^{R P A}=-2 \delta_{h h^{\prime}}\left(\epsilon\left(h-\frac{1}{2}\right)+\frac{G}{2}\right)+G,
\end{aligned}
$$

are obtained. They can be recovered from the r-RPA matrices (60) taking the additional limit

$$
\left\langle M_{i}\right\rangle \rightarrow 0
$$

\section{SUM RULES}

Given that the present RPA formalism is number conserving, we know that

$$
N|R P A\rangle=\Omega|R P A\rangle, \quad \text { with } N=\sum_{h} N_{h}+\sum_{p} N_{p},
$$

which satisfy

$$
\langle N\rangle=\sum_{h=1}^{\Omega / 2}\left(2-\left\langle M_{h}\right\rangle\right)+\sum_{p=1}^{\Omega / 2}\left\langle M_{p}\right\rangle=\Omega+\sum_{i=1}^{\Omega / 2}\left(\left\langle M_{p=i}\right\rangle-\left\langle M_{h=i}\right\rangle\right)=\Omega
$$

due to (31).

Given that

$$
\begin{aligned}
\Omega^{2}=\left\langle N^{2}\right\rangle= & \left\langle\left(\Omega+\sum_{i}\left(M_{p=i}-M_{h=i}\right)\right)\left(\Omega+\sum_{j}\left(M_{p=j}-M_{h=j}\right)\right)\right\rangle \\
= & \Omega^{2}+2 \Omega \sum_{i}\left(M_{p=i}-M_{h=i}\right)+\sum_{i j}\left(\left\langle M_{p=i} M_{p=j}\right\rangle+\left\langle M_{h=i} M_{h=j}\right\rangle\right. \\
& \left.-\left\langle M_{p=i} M_{h=j}\right\rangle-\left\langle M_{h=i} M_{h=j}\right\rangle\right)
\end{aligned}
$$

we get an additional condition over the expectation values, which is

$$
\sum_{p p^{\prime}}\left\langle M_{p} M_{p^{\prime}}\right\rangle=\sum_{h p}\left\langle M_{h} M_{p}\right\rangle
$$

This sum rule plays an important role in the discussions of the following section.

\section{THE SCRPA GROUND STATE}

In all our previous derivations we always made use of the vacuum condition (28) for the ground state $|S C R P A\rangle$ of the present theory. However, at no step have we constructed the ground state explicitly. This situation is in fact quite common. Also Hartree-Fock and Hartree-Fock-Bogoliubov theories can be derived using analogous vacuum conditions, without constructing the ground state wave function explicitly (in spite of the fact that their construction is perfectly possible). The question then naturally arises whether from (28) it is possible to explicitly construct the SCRPA ground state. 
To this purpose let us frst consider the $\Omega=2$ case. In this case there is only one particle state $(p)$ and one hole state $(h)$. There are two unperturbed (basis) states with 2 particles. They are

$$
|h\rangle=|H F\rangle \quad \text { and } \quad|p\rangle=Q_{p}^{\dagger} Q_{h}^{\dagger}|H F\rangle .
$$

The frst state has the two particles in the lower state $(h)$, the second in the upper state $(p)$.

The SCRPA ground state can be expanded in this basis as

$$
|S C R P A\rangle=a_{h}|h\rangle+a_{p}|p\rangle \quad \text { with } \quad a_{h}^{2}+a_{p}^{2}=1 .
$$

The coeff cients $a_{h}, a_{p}$ must be determined from the vacuum condition (28), which in this simple case reads

$$
A|S C R P A\rangle=\left(X_{p} \bar{Q}_{p}^{\dagger}-Y_{h} \bar{Q}_{h}\right)|S C R P A\rangle=0 .
$$

In this case $X_{p}=X_{h}=X$ and $Y_{p}=Y_{h}=Y$, and the removal operator $R$ acting on the SCRPA vacuum yields the same equation, which is

$$
X a_{p}-Y a_{h}=0
$$

Together with the normalization condition, they have the solutions

$$
a_{h}=\frac{X}{\sqrt{X^{2}+Y^{2}}}, \quad a_{p}=\frac{Y}{\sqrt{X^{2}+Y^{2}}} .
$$

In this way the SCRPA ground state is entirely determined by the RPA amplitudes X, Y. In the following section it will in fact be shown that the SCRPA can reproduce exactly the correlation and excitation energies in the case of $\Omega=2$.

Let us now consider the $\Omega=4$ case. The phonon operators for the addition mode are

$$
A_{\mu}^{\dagger}=\sum_{p=1}^{2} X_{p}^{\mu} \bar{Q}_{p}^{\dagger}-\sum_{h=1}^{2} Y_{h}^{\mu} \bar{Q}_{h}=\sum_{p=1}^{2} \tilde{X}_{p}^{\mu} Q_{p}^{\dagger}-\sum_{h=1}^{2} \tilde{Y}_{h}^{\mu} Q_{h}
$$

where we have introduced the shorthand notation

$$
\tilde{X}_{p}^{\mu}=X_{p}^{\mu} / \sqrt{1-\left\langle M_{p}\right\rangle}, \quad \tilde{Y}_{h}^{\mu}=Y_{h}^{\mu} / \sqrt{1-\left\langle M_{h}\right\rangle} .
$$

The SCRPA vacuum for 4 particles states has the form

$$
|S C R P A\rangle=a_{0}|H F\rangle+\sum_{h p} a_{h p} Q_{h}^{\dagger} Q_{p}^{\dagger}|H F\rangle+a_{2} Q_{h_{1}}^{\dagger} Q_{h_{2}}^{\dagger} Q_{p_{1}}^{\dagger} Q_{p_{2}}^{\dagger}|H F\rangle .
$$

The six coeff cients $a_{0}, a_{h p}, a_{2}$ must be determined using the normalization and the vacuum conditions. They yield to the equations

$$
\sum_{p} \tilde{X}_{p}^{\mu} a_{h p}-\tilde{Y}_{h}^{\mu} a_{0}=0, \quad \tilde{X}_{p}^{\mu} a_{2}-\sum_{h} \tilde{Y}_{h}^{\mu} a_{h p}=0 .
$$

There are four of these equations ( 2 for $h=1,2$ and 2 for $p=1,2$ ) for each of the 2 possible $\mu$ 's, giving a total of 8 equations, plus the normalization condition, to obtain the $6 a$ 's.

These equations are consistent with the simplest HF $(G=0)$ limit. In this case

$$
\tilde{X}_{p}^{\mu}=\delta_{\mu p}, \quad \tilde{Y}_{h}^{\mu}=0, \quad \mu, p, h=1,2
$$


From Eq. (78) we get $a_{h p}=0$ for all $h, p$, and $a_{2}=0$. The only remaining variable to be determined is $a_{0}=1.0$ from the normalization condition. The HF ground state is recovered as expected.

Given that the $\tilde{X}_{p}^{\mu}$, s are always f nite, we can obtain four different expressions for $a_{2}$. They are

$$
a_{2}=\frac{\tilde{Y}_{1}^{1}}{\tilde{X}_{2}^{1}} a_{11}+\frac{\tilde{Y}_{2}^{1}}{\tilde{X}_{2}^{1}} a_{21}=\frac{\tilde{Y}_{1}^{1}}{\tilde{X}_{1}^{1}} a_{12}+\frac{\tilde{Y}_{2}^{1}}{\tilde{X}_{1}^{1}} a_{22}=\frac{\tilde{Y}_{1}^{2}}{\tilde{X}_{2}^{2}} a_{11}+\frac{\tilde{Y}_{2}^{2}}{\tilde{X}_{2}^{2}} a_{21}=\frac{\tilde{Y}_{1}^{2}}{\tilde{X}_{1}^{2}} a_{12}+\frac{\tilde{Y}_{2}^{2}}{\tilde{X}_{1}^{2}} a_{22} .
$$

They imply

$$
\frac{\tilde{Y}_{1}^{1}}{\tilde{X}_{1}^{1}}=\frac{\tilde{Y}_{1}^{2}}{\tilde{X}_{1}^{2}}, \quad \frac{\tilde{Y}_{2}^{1}}{\tilde{X}_{1}^{1}}=\frac{\tilde{Y}_{2}^{2}}{\tilde{X}_{1}^{2}}, \quad \frac{\tilde{Y}_{1}^{1}}{\tilde{X}_{2}^{1}}=\frac{\tilde{Y}_{1}^{2}}{\tilde{X}_{2}^{2}}, \quad \frac{\tilde{Y}_{2}^{1}}{\tilde{X}_{2}^{1}}=\frac{\tilde{Y}_{2}^{2}}{\tilde{X}_{2}^{2}}
$$

In general there is no reason for relations (81) to be fulf lled. Indeed, in numerical examples they are not satisf ed for any $\mathrm{f}$ nite value of $G$. For larger spaces the situation does not improve. It implies that besides for $\Omega=2$, the SCRPA ground state def ned by Eq. (28) does not in fact exist. However, the theory is perfectly consistent in itself and (28) must therefore be interpreted as an auxiliary relation which allows us to express expectation values such as in (29) and (30) in a well def ned way in terms of the RPA amplitudes.

In fact, using, for example, the Green's function method, as described in [13], one can obtain expressions (29), (30) via the residua of the Green's functions without ever using the vacuum condition (28) explicitly. On the other hand, the non-existence of $|S C R P A\rangle$ entails that we are not assured that our theory yields an upper bound of the ground state energy as in a truly Raleigh-Ritz variational principle. It also implies that our theory eventually contains some violation of the Pauli principle, in spite of the fact that we never made use of any boson approximation and always fully respected the commutation laws of the Fermi pair operators. In the next section, where we present the numerical results, we will return and further discuss some consequences of the non-existence of the SCRPA vacuum.

\section{NUMERICAL SOLUTIONS}

In the present section the picket fence model is solved using the fully self-consistent RPA for a different number of levels $\Omega$. The ground state and excited states energies, as well as the particle numbers and correlation functions, are compared with the exact ones, and with those obtained from the $\mathrm{SCRPA}_{1}$, the r-RPA, and the RPA. For $\Omega=2$ analytical results are presented. In all the cases we $\mathrm{f} x$ the energy scale by setting $\epsilon=1$.

\subsection{The $\Omega=2$ Case}

This case was introduced in the previous section. The Fermi level is at the energy

$$
\lambda=\frac{3}{2}-\frac{G}{2}
$$

Exact correlated states are built as linear combinations of the states basis states $|h\rangle$ and $|p\rangle$,

$$
|\alpha\rangle=c_{h}|h\rangle+c_{p}|p\rangle \quad \text { with } c_{p}^{2}+c_{h}^{2}=1,
$$

which satisfy the eigenvalue equation

$$
\left(\begin{array}{cc}
-1 & G \\
G & 1
\end{array}\right)\left(\begin{array}{l}
c_{h} \\
c_{p}
\end{array}\right)=E_{\alpha}\left(\begin{array}{l}
c_{h} \\
c_{p}
\end{array}\right)
$$


with solutions

$$
E_{ \pm}= \pm \sqrt{1+G^{2}}, \quad c_{h}=\frac{G}{\sqrt{G^{2}+\left(E_{ \pm}+1\right)^{2}}}, \quad c_{p}=\frac{E_{ \pm}+1}{\sqrt{G^{2}+\left(E_{ \pm}+1\right)^{2}}} .
$$

The exact ground state $|0\rangle$ has energy $E_{-}=-\sqrt{1-G^{2}}$. The correlation energy $E_{\text {corr }}$ is

$$
E_{\text {corr }}=\langle 0|H| 0\rangle-\langle H F|H| H F\rangle=-\sqrt{1+G^{2}}+1 .
$$

The exact particle number expectation values are

$$
\left\langle 0\left|M_{h}\right| 0\right\rangle=\left\langle 0\left|M_{p}\right| 0\right\rangle=2 a_{p}
$$

and the exact correlation functions are

$$
\left\langle 0\left|M_{h} M_{h}\right| 0\right\rangle=\left\langle 0\left|M_{p} M_{p}\right| 0\right\rangle=\left\langle 0\left|M_{p} M_{h}\right| 0\right\rangle=4 a_{p} .
$$

The addition mode has 4 particles and only one state

$$
|4\rangle=Q_{p}^{\dagger}|H F\rangle,
$$

with

$$
\langle 4|H| 4\rangle=0 .
$$

The exact excitation energy associated to this mode is

$$
E_{\mathrm{exc}}=\langle 4|H| 4\rangle-\langle 0|H| 0\rangle=-E_{-}=\sqrt{1+G^{2}} .
$$

The SCRPA equations for the addition mode are

$$
\left(\begin{array}{cc}
1-2 G X Y & G\left(1+2 Y^{2}\right) \\
-G\left(1+2 Y^{2}\right) & -1+2 G X Y
\end{array}\right)\left(\begin{array}{c}
X \\
Y
\end{array}\right)=E\left(\begin{array}{c}
X \\
Y
\end{array}\right)
$$

with solutions

$$
E_{1,2}= \pm \sqrt{1+G^{2}}, \quad X=\frac{G}{\sqrt{G^{2}-\left(1-E_{1,2}\right)^{2}}}, \quad Y=\frac{1-E_{1,2}}{\sqrt{G^{2}-\left(1-E_{1,2}\right)^{2}}} .
$$

The addition mode has excitation energy $E_{1}$, which is exactly the same as $E_{\text {exc }}$ deduced above, although the expressions for $X, Y$ are quite different from those obtained for $a_{1}, a_{2}$.

The occupation numbers are

$$
\left\langle M_{p}\right\rangle=\left\langle M_{h}\right\rangle=1-\frac{1}{1+2 Y^{2}}=2 \frac{\left(1-E_{1}\right)^{2}}{G^{2}-\left(1-E_{1}\right)^{2}}
$$

which again reproduce the exact results.

The standard RPA equations for the addition mode are

$$
\left(\begin{array}{cc}
1 & G \\
-G & -1
\end{array}\right)\left(\begin{array}{l}
X \\
Y
\end{array}\right)=E_{R P A}\left(\begin{array}{l}
X \\
Y
\end{array}\right)
$$




\section{TABLE I}

Correlation Energies as a Function of $G$ for $\Omega=2$ Obtained with an Exact Calculation, with the RPA, r-RPA, $\mathrm{SCRPA}_{1}$, and SCRPA Methods

\begin{tabular}{lccccc}
\hline$G$ & Exact & RPA & r-RPA & SCRPA $_{1}$ & SCRPA \\
\hline 0.0 & 0.0 & 0.0 & 0.0 & 0.0 & 0.0 \\
0.2 & -0.0198 & -0.0202 & -0.0198 & -0.0198 & -0.0198 \\
0.4 & -0.0770 & -0.0835 & -0.0770 & -0.0755 & -0.0770 \\
0.6 & -0.1661 & -0.2000 & -0.1362 & -0.1568 & -0.1661 \\
0.8 & -0.2806 & -0.4000 & -0.2065 & -0.2532 & -0.2806 \\
0.90 & -0.3454 & -0.5641 & -0.2415 & -0.3050 & -0.3454 \\
\hline
\end{tabular}

with solutions

$$
E_{R P A}= \pm \sqrt{1-G^{2}}
$$

The RPA excitation energy exhibits the well known "collapse" when $G=1$, and is a decreasing function of $G$, while the exact energy is an increasing function.

The renormalized RPA (r-RPA) equations for the addition mode are

$$
\left(\begin{array}{cc}
1+G \frac{2 Y^{2}}{1+2 Y^{2}} & G \frac{1}{1+2 Y^{2}} \\
-G \frac{1}{1+2 Y^{2}} & -1-G \frac{2 Y^{2}}{1+2 Y^{2}}
\end{array}\right)\left(\begin{array}{c}
X \\
Y
\end{array}\right)=E_{r-R P A}\left(\begin{array}{c}
X \\
Y
\end{array}\right)
$$

Its solutions are not analytically simple. Numerical results can be seen in Tables I and II together with the energies obtained with the other formalisms and the exact solutions.

The contrast with the standard RPA exhibits the power of the fully self consistent RPA formalism, which in the $\Omega=2$ case was shown to reproduce the exact results.

\subsection{The $\Omega=4$ Case}

We label the four levels, from bottom to top, as $h_{2}, h_{1}, p_{1}, p_{2}$. The exact eigenstates for 4 particles states, including the ground state, are

$$
|\alpha\rangle=c_{0}^{\alpha}|H F\rangle+\sum_{h p} c_{h p}^{\alpha} Q_{h}^{\dagger} Q_{p}^{\dagger}|H F\rangle+c_{2}^{\alpha} Q_{h_{1}}^{\dagger} Q_{h_{2}}^{\dagger} Q_{p_{1}}^{\dagger} Q_{p_{2}}^{\dagger}|H F\rangle
$$

TABLE II

Energies of the First Excited State as a Function of $G$ for $\Omega=2$ Obtained with an Exact Calculation, with the RPA, r-RPA, SCRPA 1 , and SCRPA Methods

\begin{tabular}{llllll}
\hline$G$ & Exact & RPA & r-RPA & SCRPA $_{1}$ & SCRPA \\
\hline 0.0 & 1.0 & 1.0 & 1.0 & 1.0 & 1.0 \\
0.2 & 1.0198 & 0.9798 & 0.9845 & 1.0045 & 1.0198 \\
0.4 & 1.0770 & 0.9165 & 0.9572 & 1.0139 & 1.0770 \\
0.6 & 1.1662 & 0.8000 & 0.9397 & 1.0227 & 1.1662 \\
0.8 & 1.2806 & 0.6000 & 0.9379 & 1.4155 & 1.2806 \\
0.90 & 1.3454 & 0.4359 & 0.9419 & 1.5013 & 1.3454 \\
\hline
\end{tabular}


The coeff cients $c_{\mu}^{\alpha}$ fulf 11 the normalization condition

$$
\sum_{\mu}\left|c_{\mu}^{\alpha}\right|^{2}=1
$$

and are obtained as the eigen vectors of

$$
\left(\begin{array}{cccccc}
-4 & -G & -G & -G & -G & 0 \\
-G & -2 & -G & -G & 0 & -G \\
-G & -G & 0 & 0 & -G & -G \\
-G & -G & 0 & 0 & -G & -G \\
-G & 0 & -G & -G & 2 & -G \\
0 & -G & -G & -G & -G & 4
\end{array}\right)\left(\begin{array}{c}
c_{0}^{\alpha} \\
c_{h_{1} p_{1}}^{\alpha} \\
c_{h_{1} p_{2}}^{\alpha} \\
c_{h_{2} p_{1}}^{\alpha} \\
c_{h_{2} p_{2}}^{\alpha} \\
c_{2}^{\alpha}
\end{array}\right)=E_{\alpha}\left(\begin{array}{c}
c_{0}^{\alpha} \\
c_{h_{1} p_{1}}^{\alpha} \\
c_{h_{1} p_{2}}^{\alpha} \\
c_{h_{2} p_{1}}^{\alpha} \\
c_{h_{2} p_{2}}^{\alpha} \\
c_{2}^{\alpha}
\end{array}\right) .
$$

The particle-hole symmetry implies that there are two degenerate unperturbed states, which are

$$
Q_{h_{1}}^{\dagger} Q_{p_{2}}^{\dagger}|H F\rangle \quad \text { and } \quad Q_{h_{2}}^{\dagger} Q_{p_{1}}^{\dagger}|H F\rangle
$$

both having zero unperturbed energies. They always contribute on equal footing to any correlated state, i.e.,

$$
c_{h_{1} p_{2}}^{\alpha}=c_{h_{2} p_{1}}^{\alpha} .
$$

The exact occupation numbers are

$$
\begin{aligned}
& \left\langle\alpha\left|M_{h}\right| \alpha\right\rangle=2\left(\left|c_{h p_{1}}^{\alpha}\right|^{2}+\left|c_{h p_{2}}^{\alpha}\right|^{2}+\left|c_{2}^{\alpha}\right|^{2}\right), \\
& \left\langle\alpha\left|M_{p}\right| \alpha\right\rangle=2\left(\left|c_{h_{1} p}^{\alpha}\right|^{2}+\left|c_{h_{2} p}^{\alpha}\right|^{2}+\left|c_{2}^{\alpha}\right|^{2}\right),
\end{aligned}
$$

which due to (102) imply $\left\langle\alpha\left|M_{h}\right| \alpha\right\rangle=\left\langle\alpha\left|M_{p=h}\right| \alpha\right\rangle$; and

$$
\begin{aligned}
\left\langle\alpha\left|M_{h} M_{h}\right| \alpha\right\rangle & =2\left\langle\alpha\left|M_{h}\right| \alpha\right\rangle, \quad\left\langle\alpha\left|M_{p} M_{p}\right| \alpha\right\rangle=2\left\langle\alpha\left|M_{p}\right| \alpha\right\rangle, \\
\left\langle\alpha\left|M_{h_{1}} M_{h_{2}}\right| \alpha\right\rangle & =4\left|c_{2}^{\alpha}\right|^{2}, \quad\left\langle\alpha\left|M_{p_{1}} M_{p_{2}}\right| \alpha\right\rangle=4\left|c_{2}^{\alpha}\right|^{2}, \\
\left\langle\alpha\left|M_{p} M_{h}\right| \alpha\right\rangle & =4\left|c_{h p}^{\alpha}\right|^{2}+4\left|c_{2}^{\alpha}\right|^{2} .
\end{aligned}
$$

The exact eigenstates for states with 6 particles (addition mode) are

$$
|\beta\rangle=\sum_{p} c_{p}^{\beta} Q_{p}^{\dagger}|H F\rangle+\sum_{h} c_{h}^{\beta} Q_{h}^{\dagger} Q_{p_{1}}^{\dagger} Q_{p_{2}}^{\dagger}|H F\rangle .
$$

The coeff cients $c_{p, h}^{\beta}$ are obtained as the eigen vectors of

$$
\left(\begin{array}{cccc}
-3 & -G & G & G \\
-G & -1 & G & G \\
G & G & 1 & -G \\
G & G & -G & 3
\end{array}\right)\left(\begin{array}{c}
c_{p_{1}}^{\beta} \\
c_{p_{2}}^{\beta} \\
c_{h_{1}}^{\beta} \\
c_{h_{2}}^{\beta}
\end{array}\right)=E_{\beta}\left(\begin{array}{c}
c_{p_{1}}^{\beta} \\
c_{p_{2}}^{\beta} \\
c_{h_{1}}^{\beta} \\
c_{h_{2}}^{\beta}
\end{array}\right) .
$$


TABLE III

Correlation Energies as a Function of $G$ for $\Omega=4$ Obtained with Exact Calculation, with the RPA, r-RPA, SCRPA ${ }_{1}$, and SCRPA Methods

\begin{tabular}{cccccc}
\hline$G$ & Exact & RPA & r-RPA & SCRPA $_{1}$ & SCRPA \\
\hline 0.0 & 0.0 & 0.0 & 0.0 & 0.0 & 0.0 \\
0.1 & -0.0123 & -0.0124 & -0.0123 & -0.0124 & -0.0124 \\
0.2 & -0.0520 & -0.0538 & -0.0510 & -0.0523 & -0.0524 \\
0.3 & -0.1225 & -0.1343 & -0.1158 & -0.1233 & -0.1248 \\
0.4 & -0.2264 & -0.2764 & -0.2009 & -0.2259 & -0.2334 \\
0.5 & -0.3644 & -0.5662 & -0.2961 & -0.3575 & -0.3795 \\
\hline
\end{tabular}

The exact excitation $E_{\mathrm{exc}}^{\beta}$ energies for the addition mode are measured against the ground state energy, i.e.,

$$
E_{\mathrm{exc}}^{\beta}=E_{\beta}-E_{\alpha=1}
$$

We have calculated the exact solutions and compared them with the solutions of the SCRPA equations. This involved solving simultaneously the SCRPA eigenvalue problem, Eqs. (19), and evaluating the number correlations by solving Eq. (41). In the following tables we present the correlation energies and the excitation energies of the $\mathrm{f}$ rst and second addition modes, obtained using four different methods: the exact results, the RPA ones, the SCRPA using $\left\langle M_{i} M_{j}\right\rangle=\left\langle M_{i}\right\rangle\left\langle M_{j}\right\rangle$ (denoted SCRPA $_{1}$ ), and the fully self-consistent RPA (SCRPA) which refers to the treatment described above.

In Tables III-V the improvement offered by the SCRPA as compared with the RPA is clearly seen. Both for the correlation and the excitation energies the RPA gives only a crude approximation, while the SCRPA results are very close to the exact ones.

The correlation energies listed in Table III are slightly better described with the $\mathrm{SCRPA}_{1}$ than with the SCRPA. It is also noticeable that both the RPA and SCRPA correlation energies are lower than the exact ones, but the $\mathrm{SCRPA}_{1}$ energies are slightly higher. The situation is very different when excitation energies are analyzed (Tables IV and V). While again the RPA energies are lower than the exact ones, both the SCRPA and SCRPA $_{1}$ energies are higher. As mentioned above, they are very close to the exact ones, but in this case the SCRPA ones are clearly the best, reproducing with high accuracy the exact results.

To obtain a deeper understanding of the above discussed results a study of the occupation numbers in the different approaches is in order. They are plotted in Fig. 1 as a function of $G$. The occupation numbers $\left\langle M_{p}\right\rangle / 2$ obtained using the exact solutions are represented by thin lines, those obtained using the RPA by small dots, the SCRPA 1 by thick lines, and the SCRPA by large dots. Upper curves display the occupations for $p=1$, lower curves for $p=2$.

TABLE IV

Excitation Energy of the First Addition Mode as a Function of $G$ for $\Omega=4$ Obtained with Exact Calculation, with the RPA, r-RPA, SCRPA 1 , and SCRPA Methods

\begin{tabular}{llllll}
\hline$G$ & Exact & RPA & r-RPA & SCRPA $_{1}$ & SCRPA \\
\hline 0.0 & 1.0 & 1.0 & 1.0 & 1.0 & 1.0 \\
0.1 & 1.0027 & 0.9864 & 0.9872 & 1.0041 & 1.0027 \\
0.2 & 1.0115 & 0.9402 & 0.9494 & 1.0233 & 1.0126 \\
0.3 & 1.0276 & 0.8493 & 0.8917 & 1.0648 & 1.0348 \\
0.4 & 1.0520 & 0.6898 & 0.8248 & 1.1279 & 1.0734 \\
0.5 & 1.0853 & 0.3745 & 0.7603 & 1.2065 & 1.1274 \\
\hline
\end{tabular}


TABLE V

Excitation Energy of the Second Addition Mode as a Function of $G$ for $\Omega=4$ Obtained with Exact Calculation, with the RPA, r-RPA, SCRPA ${ }_{1}$, and SCRPA Methods

\begin{tabular}{llllll}
\hline$G$ & Exact & RPA & r-RPA & SCRPA $_{1}$ & SCRPA \\
\hline 0.0 & 3.0 & 3.0 & 3.0 & 3.0 & 3.0 \\
0.1 & 3.0104 & 3.0012 & 3.0014 & 3.0108 & 3.0104 \\
0.2 & 3.0465 & 3.0060 & 3.0075 & 3.0499 & 3.0468 \\
0.3 & 3.1156 & 3.0164 & 3.0212 & 3.1259 & 3.1168 \\
0.4 & 3.2237 & 3.0338 & 3.0446 & 3.2422 & 3.2256 \\
0.5 & 3.3738 & 3.0594 & 3.0784 & 3.3967 & 3.3784 \\
\hline
\end{tabular}

As it is well known, RPA calculations predict far more correlations than there are found in the exact ground state. For both states this is evident here: the small dotted curves increase as a function of $G$ faster then the exact ones, and diverge close to the point of collapse of the RPA. On the other hand, we learn from Fig. 1 that the SCRPA underpredict the ground state correlations. The SCRPA occupations deviate from the exact ones nearly as much as the RPA occupations, but underestimating them. It is remarkable that the SCRPA 1 energies are so good, given these discrepancies. The SCRPA occupations are very close to the exact ones.

The correlation functions $\left\langle M_{i} M_{j}\right\rangle$ are shown in Table VI for $G=0.4$.

As can be seen in Table VI the occupation number correlations obtained self-consistently from the SCRPA equations are very close to the exact ones, even for a value of $G$ as large as 0.4 . On the other hand the approximation $\left\langle M_{i} M_{j}\right\rangle \approx\left\langle M_{i}\right\rangle\left\langle M_{j}\right\rangle$ underestimates the correlations. It could explain the small occupation numbers obtained when this approximation is used in the SCRPA equations.

Although the SCRPA results presented here are in general extremely close to the exact results, considering correlation or excitation energies, occupation or correlation numbers, there is a caveat which must be mentioned here. It concerns the sum rule (69).

The sums of correlation numbers obtained using the exact calculation and the SCRPA one are very similar. They are shown in Table VII. However, while the exact results fulf 11 the sum rule (69), the SCRPA do not fulf 11 this sum rule. This shortcoming certainly stems from the fact that, as we explained in Section 8, the SCRPA ground state as def ned in Eq. (28) only exists for the special case $\Omega=2$. In all the other cases the use of relation (28) implies some degree of approximation entailing, for example, the violation of the above sum rule.

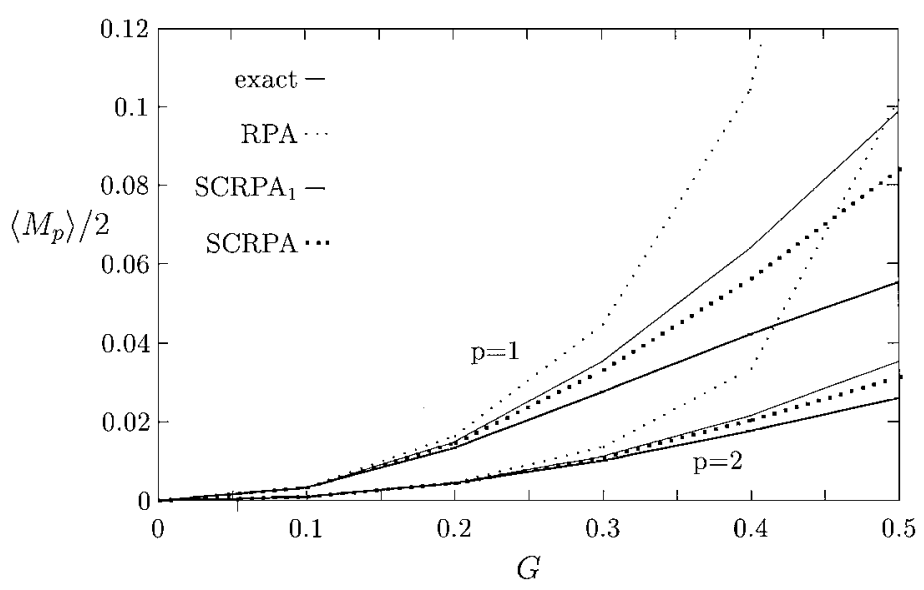

FIG. 1. Occupation numbers $\left\langle M_{p}\right\rangle / 2$ as a function of $G$ for $\Omega=4$. 
TABLE VI

Correlation Functions $\left\langle M_{i} M_{j}\right\rangle$ for $G=0.4, \Omega=4$

\begin{tabular}{ccccc}
\hline$i$ & $j$ & $\left\langle M_{h_{i}} M_{h_{j}}\right\rangle$ & $\left\langle M_{h_{i}} M_{p_{j}}\right\rangle$ & $\left\langle M_{p_{i}} M_{p_{j}}\right\rangle$ \\
\hline \multicolumn{4}{c}{ (a) Exact solutions } \\
1 & 1 & 0.2556 & 0.1967 & 0.2556 \\
1 & 2 & 0.0028 & 0.0616 & 0.0028 \\
2 & 1 & 0.0028 & 0.0616 & 0.0028 \\
2 & 2 & 0.0864 & 0.0275 & 0.0864 \\
\multicolumn{5}{c}{ (b) SCRPA solutions } \\
1 & 1 & 0.2251 & 0.1774 & 0.2251 \\
1 & 2 & 0.0033 & 0.0603 & 0.0033 \\
2 & 1 & 0.0033 & 0.0603 & 0.0033 \\
2 & 2 & 0.0816 & 0.0281 & 0.0816 \\
& & \multicolumn{4}{c}{ (c) Product matrix } \\
1 & 1 & 0.0127 & 0.0127 & 0.0127 \\
1 & 2 & 0.0046 & 0.0046 & 0.0046 \\
2 & 1 & 0.0046 & 0.0046 & 0.0046 \\
2 & 2 & 0.0017 & 0.0017 & 0.0017 \\
\hline
\end{tabular}

Note. (a) The exact results, (b) the SCRPA results, and (c) the product of single occupations.

\subsection{The $\Omega=10$ Case}

The results for $\Omega=10$ repeat the general patterns found for $\Omega=4$. In Table VIII the ground state correlation energies are presented as a function of the pairing strength $G$; in Tables IX and $\mathrm{X}$ the excitation energies of the $\mathrm{f}$ rst and second addition mode in a system with $\mathrm{N}=12$ fermions, measured relative to the ground state of the system with $\mathrm{N}=10$, are shown, respectively.

The SCRPA does not show any collapse, and the energies are far closer to the exact energies than the RPA ones. For the ground state correlation energy the ones obtained with the $\mathrm{SCRPA}_{1}$ formalism are slightly closer to the exact ones than the SCRPA energies, while the opposite is true for the $\mathrm{frst}$ and second excited states. For these excitation energies the SCRPA reproduces with very high accuracy the exact values. It is worth mentioning that the exact excitation energies of the $\mathrm{f}$ rst and second addition modes monotonically increase as a function of $G$. This behavior is well reproduced by the SCRPA, while in the standard RPA these excitation energies are decreasing. This implies that the screening of the bare interaction is so strong that even the sign of the interaction is turned around, bringing the SCRPA solution closer to the exact one. This is a quite remarkable achievement of SCRPA.

The sum rule (69) is again violated in the $\Omega=10$ case, as shown in Table XI. It must be said that the difference between both sums, of the order 0.02 , must be compared with $N^{2}=100$. The violation of the sum rule is therefore very small. On the other side, it exhibits the limitations of the best possible SCRPA treatment of this problem.

TABLE VII

Sum Rule (69) for the Exact and SCRPA Calculations for $G=0.4, \Omega=4$

\begin{tabular}{lcc}
\hline & $\sum_{p p^{\prime}}\left\langle M_{p} M_{p^{\prime}}\right\rangle$ & $\sum_{h p}\left\langle M_{h} M_{p}\right\rangle$ \\
\hline Exact & 0.3476 & 0.3476 \\
SCRPA & 0.3133 & 0.3260 \\
\hline
\end{tabular}


TABLE VIII

Correlation Energies as a Function of $G$ Obtained with Exact Calculation, with the RPA, SCRPA 1 , and SCRPA Methods, for $\Omega=10$

\begin{tabular}{lccrr}
\hline$G$ & Exact & RPA & SCRPA $_{1}$ & SCRPA \\
\hline .00 & 0.0000 & 0.0000 & 0.0000 & 0.0000 \\
.05 & -0.0086 & -0.0086 & -0.0086 & -0.0086 \\
.10 & -0.0364 & -0.0367 & -0.0365 & -0.0365 \\
.20 & -0.1669 & -0.1756 & -0.1686 & -0.1691 \\
.30 & -0.4350 & -0.5419 & -0.4424 & -0.4497 \\
.33 & -0.5505 & -0.8181 & -0.5594 & -0.5725 \\
.34 & -0.5931 & $* * *$ & -0.6023 & -0.6180 \\
.35 & -0.6379 & $* * *$ & -0.6473 & -0.6658 \\
.36 & -0.6850 & $* * *$ & -0.6943 & -0.7160 \\
\hline
\end{tabular}

TABLE IX

Excitation Energy of the First Addition Mode as a Function of $G$ Obtained with Exact Calculation, with the RPA, SCRPA $_{1}$, and SCRPA Methods, for $\Omega=10$

\begin{tabular}{lcccr}
\hline$G$ & Exact & RPA & SCRPA $_{1}$ & SCRPA \\
\hline .00 & 1.0000 & 1.0000 & 1.0000 & 1.0000 \\
.05 & 1.0003 & 0.9940 & 1.0005 & 1.0003 \\
.10 & 1.0011 & 0.9732 & 1.0034 & 1.0014 \\
.20 & 1.0053 & 0.8604 & 1.0279 & 1.0119 \\
.30 & 1.0143 & 0.5257 & 1.0970 & 1.0539 \\
.33 & 1.0184 & 0.2574 & 1.1266 & 1.0758 \\
.34 & 1.0199 & $* * *$ & 1.1372 & 1.0840 \\
.35 & 1.0216 & $* * *$ & 1.1481 & 1.0927 \\
.36 & 1.0233 & $* * *$ & 1.1592 & 1.1018 \\
\hline
\end{tabular}

TABLE $X$

Excitation Energy of the Second Addition Mode as a Function of $G$ Obtained with Exact Calculation, with the RPA, SCRPA 1 , and SCRPA Methods, for $\Omega=10$

\begin{tabular}{lcccr}
\hline$G$ & Exact & RPA & SCRPA $_{1}$ & SCRPA \\
\hline .00 & 3.0000 & 3.0000 & 3.0000 & 3.0000 \\
.05 & 3.0010 & 2.9971 & 3.0011 & 3.0010 \\
.10 & 3.0056 & 2.9881 & 3.0063 & 3.0057 \\
.20 & 3.0390 & 2.9515 & 3.0460 & 3.0406 \\
.30 & 3.1442 & 2.8935 & 3.1657 & 3.1507 \\
.33 & 3.1998 & 2.8736 & 3.2244 & 3.2070 \\
.34 & 3.2214 & $* * *$ & 3.2467 & 3.2287 \\
.35 & 3.2449 & $* * *$ & 3.2703 & 3.2518 \\
.36 & 3.2701 & $* * *$ & 3.2952 & 3.2765 \\
\hline
\end{tabular}

TABLE XI

Sum Rule (69) for the Exact and SCRPA Calculations for $G=0.3, \Omega=10$

\begin{tabular}{lcc}
\hline & $\sum_{p p^{\prime}}\left\langle M_{p} M_{p^{\prime}}\right\rangle$ & $\sum_{h p}\left\langle M_{h} M_{p}\right\rangle$ \\
\hline Exact & 0.5173 & 0.5173 \\
SCRPA & 0.4509 & 0.4736 \\
\hline
\end{tabular}




\section{TABLE XII}

Correlation Energies as a Function of $G$ Obtained with Exact Calculation, with the RPA, r-RPA, $\mathrm{SCRPA}_{1}$, and SCRPA Methods for $\Omega=24$

\begin{tabular}{rrrrrr}
\hline$G$ & Exact & \multicolumn{1}{c}{ RPA } & r-RPA & SCRPA $_{1}$ & SCRPA \\
\hline 0.00 & 0.0000 & 0.0000 & 0.0000 & 0.0000 & 0.0000 \\
0.05 & -0.0218 & -0.0218 & -0.0218 & -0.0218 & -0.0218 \\
0.10 & -0.0953 & -0.0961 & -0.0955 & -0.0955 & -0.0955 \\
0.15 & -0.2373 & -0.2432 & -0.2358 & -0.2386 & -0.2388 \\
0.20 & -0.4736 & -0.5096 & -0.4623 & -0.4794 & -0.4809 \\
0.25 & -0.8452 & -1.1420 & -0.7808 & -0.8607 & -0.8694 \\
\hline
\end{tabular}

TABLE XIII

Excitation Energy of the First Addition Mode as a Function of $G$ Obtained with the RPA, r-RPA, $\mathrm{SCRPA}_{1}$, and SCRPA Methods for $\Omega=24$

\begin{tabular}{ccccc}
\hline$G$ & RPA & r-RPA & SCRPA $_{1}$ & SCRPA \\
\hline 0.00 & 1.0000 & 1.0000 & 1.0000 & 1.0000 \\
0.05 & 0.9911 & 0.9912 & 1.0004 & 1.0001 \\
0.10 & 0.9574 & 0.9594 & 1.0035 & 1.0011 \\
0.15 & 0.8806 & 0.8936 & 1.0153 & 1.0060 \\
0.20 & 0.7155 & 0.7818 & 1.0458 & 1.0233 \\
0.25 & 0.2132 & 0.6279 & 1.1040 & 1.0661 \\
\hline
\end{tabular}

\section{TABLE XIV}

Excitation Energy of the second Addition Mode as a Function of $G$ Obtained with the RPA, r-RPA, $\mathrm{SCRPA}_{1}$, and SCRPA Methods for $\Omega=24$

\begin{tabular}{ccccc}
\hline$G$ & RPA & r-RPA & SCRPA $_{1}$ & SCRPA \\
\hline 0.00 & 3.0000 & 3.0000 & 3.0000 & 3.0000 \\
0.05 & 2.9944 & 2.9944 & 3.0007 & 3.0006 \\
0.10 & 2.9755 & 2.9761 & 3.0057 & 3.0049 \\
0.15 & 2.9398 & 2.9425 & 3.0233 & 3.0200 \\
0.20 & 2.8836 & 2.8926 & 3.0705 & 3.0622 \\
0.25 & 2.8044 & 2.8297 & 3.1758 & 3.1618 \\
\hline
\end{tabular}

TABLE XV

Correlation Energies as a Function of $G$ Obtained with Exact Calculation, with the RPA, r-RPA, and $\mathrm{SCRPA}_{1}$ Methods for $\Omega=100$

\begin{tabular}{ccccr}
\hline$G$ & Exact & RPA & r-RPA & SCRPA $_{1}$ \\
\hline 0.00 & 0.0000 & 0.0000 & 0.0000 & 0.0000 \\
0.05 & -0.0946 & -0.0946 & -0.0946 & -0.0946 \\
0.10 & -0.4250 & -0.4274 & -0.4247 & -0.4256 \\
0.12 & -0.6467 & -0.6540 & -0.6454 & -0.6483 \\
0.14 & -0.9365 & -0.9575 & -0.9314 & -0.9405 \\
0.16 & -1.3127 & -1.3768 & -1.2942 & -1.3217 \\
0.17 & -1.5413 & -1.6628 & -1.5067 & -1.5543 \\
0.18 & -1.8027 & -2.0914 & -1.7395 & -1.8209 \\
0.19 & -2.1028 & $* * *$ & $* * *$ & -2.1273 \\
\hline
\end{tabular}


TABLE XVI

Excitation Energy of the First Addition Mode as a Function of $G$ Obtained with the RPA, r-RPA, and $\mathrm{SCRPA}_{1}$ Methods for $\Omega=100$

\begin{tabular}{cccc}
\hline$G$ & RPA & r-RPA & SCRPA $_{1}$ \\
\hline 0.00 & 1.0000 & 1.0000 & 1.0000 \\
0.05 & 0.9857 & 0.9859 & 1.0004 \\
0.10 & 0.9210 & 0.9252 & 1.0056 \\
0.12 & 0.8657 & 0.8774 & 1.0126 \\
0.14 & 0.7762 & 0.8087 & 1.0262 \\
0.16 & 0.6186 & 0.7144 & 1.0504 \\
0.17 & 0.4829 & 0.6575 & 1.0678 \\
0.18 & 0.2181 & 0.5958 & 1.0895 \\
0.19 & $* * *$ & $* * *$ & 1.1154 \\
\hline
\end{tabular}

\subsection{The $\Omega=24$ and $\Omega=100$ Cases}

The correlation energies for $\Omega=24$ are shown in Table XII. The excitation energies corresponding to the frst and second addition modes are presented in Tables XIII and XIV, respectively. All the energies are shown as functions of the pairing strength $G$ given in the f rst columns. The second column exhibits the exact results in Table XII. RPA, r-RPA, SCRPA 1 , and SCRPA energies are presented in the following four columns.

The large $\Omega$ limit is being approached, where all the RPA descriptions show their best performances. For the correlation energies even the standard RPA predictions are good, except for $G=0.25$, close to the point of collapse. For the excitation energies both the RPA and r-RPA results decrease when $G$ increases, while the SCRPA energies are increasing, as in previous cases, and in agreement with the exact energies (not shown). At the same time the differences between the $\mathrm{SCRPA}_{1}$ and SCRPA energies are vanishing. It strongly supports the use of the simpler SCRPA approach for larger $\Omega$, where the numerical effort needed to solve the SCRPA equations in the fully self-consistent formalism is not justif ed.

This is the case for $\Omega=100$, whose energies are shown in Tables XV-XVII as functions of the pairing strength $G$. Table XV exhibits the exact correlation energies in the second columns, and the RPA, r-RPA, and SCRPA 1 in the third, fourth, and $\mathrm{f} f$ th columns, respectively. The agreement between the exact and SCRPA is excellent, even for $G=0.19$, where the RPA has collapsed.

Tables XVI and XVII present the RPA, r-RPA, and SCRPA $_{1}$ excitation energies of the frst and second addition modes as a function of $G$. Again only the SCRPA energies are increasing when $G$

TABLE XVII

Excitation Energy of the Second Addition Mode as a Function of $G$ Obtained with the RPA, r-RPA, and $\mathrm{SCRPA}_{1}$ Methods for $\Omega=100$

\begin{tabular}{cccc}
\hline$G$ & RPA & r-RPA & SCRPA $_{1}$ \\
\hline 0.00 & 3.0000 & 3.0000 & 3.0000 \\
0.05 & 2.9896 & 2.9897 & 3.0006 \\
0.10 & 2.9489 & 2.9499 & 3.0082 \\
0.12 & 2.9193 & 2.9218 & 3.0182 \\
0.14 & 2.8788 & 2.8843 & 3.0381 \\
0.16 & 2.8245 & 2.8362 & 3.0761 \\
0.17 & 2.7912 & 2.8080 & 3.1059 \\
0.18 & 2.7530 & 2.7773 & 3.1457 \\
0.19 & $* * *$ & $* * *$ & 3.1985 \\
\hline
\end{tabular}


increases, as is expected from the exact results. The latter results are not shown, since it would have needed extra numerical effort without bringing new insight to the problem. It should be mentioned that $\Omega=100$ constitutes an extremely large conf guration space which by no means can be handled with ordinary diagonalization procedures.

It is well known that the RPA collapse ref ects a qualitative change in the mean f eld, which after the collapse is dominated by superf uid correlations [24]. It must be remembered that, while the energetics and the occupation numbers obtained with the SCRPA are very close to the exact ones, the wave functions around and beyond the value of $G$ at which standard RPA collapses, being far better than those obtained with RPA or r-RPA, can nonetheless have an overlap with the exact wave function of less than 50\% [25]. In this case the SCRPA must be extended to the deformed, i.e., superf uid, basis [11].

\section{CONCLUSIONS}

In this work we presented for the frst time a fully self-consistent RPA treatment of the picket fence model. This involved developing equations for some correlation functions which had to be solved simultaneously with the SCRPA eigenvalue problem.

One of the remarkable results of the present work is that for $\Omega=2$, i.e., for the 2 particles case, SCRPA provides the exact energies of the problem. This is not at all trivial because usually many body approaches deteriorate as the number of particles decreases. For higher number of levels SCRPA is, of course, not any longer exact but the results for the correlation energy and the low lying part of the spectrum are in excellent agreement with the exact ones.

In an earlier work we had used the factorization ansatz $\left\langle N_{i} N_{j}\right\rangle \approx\left\langle N_{i}\right\rangle\left\langle N_{j}\right\rangle$ for the density-density correlation functions. As mentioned already, we did not make any of these approximations in the present work. Nevertheless we could verify a posteriori that the above approximation yields still excellent results as compared with the exact solutions. This is more noticeable when $\Omega$ is increased. Actually we found that the above factorization approximation also works well for other models [16]. Therefore one may conjecture that it is quite generally valid. It is also quite useful, since it reduces the numerical work considerably. For example, with its use we solved the SCRPA equations for $\Omega=100$ without problems.

In spite of the excellent results we could not conf rm previous conjectures, at least for the ansatz used in this work, that SCRPA yields an upper limit to the ground state energy. Our values are very accurate but slightly below the exact values. This f nding is certainly linked to the fact that for the present case a self-consistent RPA ground state wave function does not exist except for $\Omega=2$, as we showed in Section 8. This most likely entails a violation of the Pauli principle which, although small, in turn gives rise to overbinding. This also explains the slight violation of the sum rule (67). One should, however, mention that the picket fence model, because of the low degeneracy of the levels, tests the Pauli principle in a most sensitive way. This is, for example, borne out in the fact that the low lying excited states, as a function of $G$, rise instead of get lower as one could expect from the attractive nature of the interaction. It is another outstanding result of the present application of the SCRPA theory that its results follow very closely this trend of the excited states implying that the renormalization of the bare interaction due to the self-consistency effect can even turn around the sign of the interaction.

We also should mention that we have treated only the non-superf uid phase. It is well known from our previous studies that for interaction strengths beyond the collapse of standard RPA one must switch to the "deformed" or superf uid basis, otherwise the results deteriorate or the iteration procedure does not converge. The superf uid domain of the present model shall be studied in a future work.

All in all we can conclude that in this frst full application of SCRPA to a large scale problem the expectation we had gained from earlier studies in the performance of SCRPA has been fully 
conf rmed. Indeed the results did not fail in any major quantitative respect. It may therefore seem worthwhile to push the applications of SCRPA to more realistic Hamiltonians.

\section{APPENDIX: SOME USEFUL RELATIONS}

$$
\begin{aligned}
& {\left[R_{\lambda}, R_{\lambda^{\prime}}^{\dagger}\right]=-\sum_{p} Y_{p}^{\lambda} Y_{p}^{\lambda^{\prime}} \frac{1-M_{p}}{1-\left\langle M_{p}\right\rangle}+\sum_{h} X_{h}^{\lambda} X_{h}^{\lambda^{\prime}} \frac{1-M_{h}}{1-\left\langle M_{h}\right\rangle},} \\
& {\left[A_{\mu}, A_{\mu^{\prime}}^{\dagger}\right]=\sum_{p} X_{p}^{\mu} X_{p}^{\mu^{\prime}} \frac{1-M_{p}}{1-\left\langle M_{p}\right\rangle}-\sum_{h} Y_{h}^{\mu} Y_{h}^{\mu^{\prime}} \frac{1-M_{h}}{1-\left\langle M_{h}\right\rangle},} \\
& {\left[A_{\mu}, R_{\lambda}\right]=-\sum_{p} X_{p}^{\mu} Y_{p}^{\lambda} \frac{1-M_{p}}{1-\left\langle M_{p}\right\rangle}+\sum_{h} Y_{h}^{\mu} X_{h}^{\lambda} \frac{1-M_{h}}{1-\left\langle M_{h}\right\rangle},} \\
& {\left[M_{p}, R_{\lambda}\right]=-2 Y_{p}^{\lambda}\left\{\sum_{\mu_{1}} X_{p}^{\mu_{1}} A_{\mu_{1}}^{\dagger}+\sum_{\lambda_{1}} Y_{p}^{\lambda_{1}} R_{\lambda_{1}}\right\}} \\
& {\left[M_{h}, A_{\mu}^{\dagger}\right]=2 Y_{h}^{\mu}\left\{\sum_{\mu_{1}} Y_{p}^{\mu_{1}} A_{\mu_{1}}^{\dagger}+\sum_{\lambda_{1}} X_{h}^{\lambda_{1}} R_{\lambda_{1}}\right\}} \\
& \left\langle R_{\lambda} R_{\lambda_{2}}^{\dagger} R_{\lambda_{1}} R_{\lambda^{\prime}}^{\dagger}\right\rangle=\sum_{p_{1} p_{2}} \frac{Y_{p_{1}}^{\lambda} Y_{p_{1}}^{\lambda_{2}}}{\left(1-\left\langle M_{p_{1}}\right\rangle\right)} \frac{Y_{p_{2}}^{\lambda_{1}} Y_{p_{2}}^{\lambda^{\prime}}}{\left(1-\left\langle M_{p_{2}}\right\rangle\right)}\left\langle\left(1-M_{p_{1}}\right)\left(1-M_{p_{2}}\right)\right\rangle \\
& -\sum_{p_{1} h_{1}} \frac{Y_{p_{1}}^{\lambda} Y_{p_{1}}^{\lambda_{2}}}{\left(1-\left\langle M_{p_{1}}\right\rangle\right)} \frac{X_{h_{1}}^{\lambda_{1}} X_{h_{1}}^{\lambda^{\prime}}}{\left(1-\left\langle M_{h_{1}}\right\rangle\right)}\left\langle\left(1-M_{p_{1}}\right)\left(1-M_{h_{1}}\right)\right\rangle \\
& -\sum_{p_{1} h_{1}} \frac{Y_{p_{1}}^{\lambda_{1}} Y_{p_{1}}^{\lambda^{\prime}}}{\left(1-\left\langle M_{p_{1}}\right\rangle\right)} \frac{X_{h_{1}}^{\lambda} X_{h_{1}}^{\lambda_{2}}}{\left(1-\left\langle M_{h_{1}}\right\rangle\right)}\left\langle\left(1-M_{p_{1}}\right)\left(1-M_{h_{1}}\right)\right\rangle \\
& +\sum_{h_{1} h_{2}} \frac{X_{h_{1}}^{\lambda} X_{h_{1}}^{\lambda_{2}}}{\left(1-\left\langle M_{h_{1}}\right\rangle\right)} \frac{X_{h_{2}}^{\lambda_{1}} X_{h_{2}}^{\lambda^{\prime}}}{\left(1-\left\langle M_{h_{2}}\right\rangle\right)}\left\langle\left(1-M_{h_{1}}\right)\left(1-M_{h_{2}}\right)\right\rangle \\
& \left\langle R_{\lambda}\left[R_{\lambda_{1}}, R_{\lambda_{2}}^{\dagger}\right] R_{\lambda^{\prime}}^{\dagger}\right\rangle=2 \sum_{p_{1}} \frac{Y_{p_{1}}^{\lambda_{1}} Y_{p_{1}}^{\lambda_{2}} Y_{p_{1}}^{\lambda} Y_{p_{1}}^{\lambda^{\prime}}}{\left(1-\left\langle M_{p_{1}}\right\rangle\right)}-2 \sum_{h_{1}} \frac{X_{h_{1}}^{\lambda_{1}} X_{h_{1}}^{\lambda_{2}} X_{h_{1}}^{\lambda} X_{h_{1}}^{\lambda^{\prime}}}{\left(1-\left\langle M_{h_{1}}\right\rangle\right)} \\
& +\sum_{p_{1} p_{2}} \frac{Y_{p_{1}}^{\lambda_{1}} Y_{p_{1}}^{\lambda_{2}}}{\left(1-\left\langle M_{p_{1}}\right\rangle\right)} \frac{Y_{p_{2}}^{\lambda} Y_{p_{2}}^{\lambda^{\prime}}}{\left(1-\left\langle M_{p_{2}}\right\rangle\right)}\left\langle\left(1-M_{p_{1}}\right)\left(1-M_{p_{2}}\right)\right\rangle \\
& -\sum_{p_{1} h_{1}} \frac{Y_{p_{1}}^{\lambda_{1}} Y_{p_{1}}^{\lambda_{2}}}{\left(1-\left\langle M_{p_{1}}\right\rangle\right)} \frac{X_{h_{1}}^{\lambda} X_{h_{1}}^{\lambda^{\prime}}}{\left(1-\left\langle M_{h_{1}}\right\rangle\right)}\left\langle\left(1-M_{p_{1}}\right)\left(1-M_{h_{1}}\right)\right\rangle \\
& -\sum_{p_{1} h_{1}} \frac{Y_{p_{1}}^{\lambda} Y_{p_{1}}^{\lambda^{\prime}}}{\left(1-\left\langle M_{p_{1}}\right\rangle\right)} \frac{X_{h_{1}}^{\lambda_{1}} X_{h_{1}}^{\lambda_{2}}}{\left(1-\left\langle M_{h_{1}}\right\rangle\right)}\left\langle\left(1-M_{p_{1}}\right)\left(1-M_{h_{1}}\right)\right\rangle \\
& +\sum_{h_{1} h_{2}} \frac{X_{h_{1}}^{\lambda_{1}} X_{h_{1}}^{\lambda_{2}}}{\left(1-\left\langle M_{h_{1}}\right\rangle\right)} \frac{X_{h_{2}}^{\lambda} X_{h_{2}}^{\lambda^{\prime}}}{\left(1-\left\langle M_{h_{2}}\right\rangle\right)}\left\langle\left(1-M_{h_{1}}\right)\left(1-M_{h_{2}}\right)\right\rangle
\end{aligned}
$$




$$
\begin{aligned}
\left\langle R_{\lambda} R_{\lambda_{1}} R_{\lambda_{2}}^{\dagger} R_{\lambda^{\prime}}^{\dagger}\right\rangle= & \left\langle R_{\lambda}\left[R_{\lambda_{1}}, R_{\lambda_{2}}^{\dagger}\right] R_{\lambda^{\prime}}^{\dagger}\right\rangle+\left\langle R_{\lambda} R_{\lambda_{1}}^{\dagger} R_{\lambda_{2}} R_{\lambda^{\prime}}^{\dagger}\right\rangle \\
= & 2 \sum_{p_{1}} \frac{Y_{p_{1}}^{\lambda_{1}} Y_{p_{1}}^{\lambda_{2}} Y_{p_{1}}^{\lambda} Y_{p_{1}}^{\lambda^{\prime}}}{\left(1-\left\langle M_{p_{1}}\right\rangle\right)}-2 \sum_{h_{1}} \frac{X_{h_{1}}^{\lambda_{1}} X_{h_{1}}^{\lambda_{2}} X_{h_{1}}^{\lambda} X_{h_{1}}^{\lambda^{\prime}}}{\left(1-\left\langle M_{h_{1}}\right\rangle\right)} \\
& +\sum_{p_{1} p_{2}} \frac{Y_{p_{1}}^{\lambda_{2}} Y_{p_{2}}^{\lambda^{\prime}}\left(Y_{p_{1}}^{\lambda} Y_{p_{2}}^{\lambda_{1}}+Y_{p_{1}}^{\lambda_{1}} Y_{p_{2}}^{\lambda}\right)}{\left(1-\left\langle M_{p_{1}}\right\rangle\right)\left(1-\left\langle M_{p_{2}}\right\rangle\right)}\left\langle\left(1-M_{p_{1}}\right)\left(1-M_{p_{2}}\right)\right\rangle \\
& -\sum_{p_{1} h_{1}} \frac{\left(Y_{p_{1}}^{\lambda_{2}} X_{h_{1}}^{\lambda^{\prime}}+Y_{p_{1}}^{\lambda^{\prime}} X_{h_{1}}^{\lambda_{2}}\right)\left(Y_{p_{1}}^{\lambda_{1}} X_{h_{1}}^{\lambda}+Y_{p_{1}}^{\lambda} X_{h_{1}}^{\lambda_{1}}\right)}{\left(1-\left\langle M_{p_{1}}\right\rangle\right)\left(1-\left\langle M_{h_{1}}\right\rangle\right)}\left\langle\left(1-M_{p_{1}}\right)\left(1-M_{p_{2}}\right)\right\rangle \\
& +\sum_{h_{1} h_{2}} \frac{X_{h_{1}}^{\lambda_{2}} X_{h_{2}}^{\lambda^{\prime}}\left(X_{h_{1}}^{\lambda_{1}} X_{h_{2}}^{\lambda}+X_{h_{1}}^{\lambda} X_{h_{2}}^{\lambda_{1}}\right)}{\left(1-\left\langle M_{h_{1}}\right\rangle\right)\left(1-\left\langle M_{h_{2}}\right\rangle\right)}\left\langle\left(1-M_{h_{1}}\right)\left(1-M_{h_{2}}\right)\right\rangle
\end{aligned}
$$

$$
\begin{aligned}
\left\langle A_{\mu} A_{\mu_{1}} A_{\mu_{2}}^{\dagger} A_{\mu^{\prime}}^{\dagger}\right\rangle= & 2 \sum_{h_{1}} \frac{Y_{h_{1}}^{\mu_{1}} Y_{h_{1}}^{\mu_{2}} Y_{h_{1}}^{\mu} Y_{h_{1}}^{\mu^{\prime}}}{\left(1-\left\langle M_{h_{1}}\right\rangle\right)}-2 \sum_{p_{1}} \frac{X_{p_{1}}^{\mu_{1}} X_{p_{1}}^{\mu_{2}} X_{p_{1}}^{\mu} X_{p_{1}}^{\mu^{\prime}}}{\left(1-\left\langle M_{p_{1}}\right\rangle\right)} \\
& +\sum_{h_{1} h_{2}} \frac{Y_{h_{1}}^{\mu_{2}} Y_{h_{2}}^{\mu^{\prime}}\left(Y_{h_{1}}^{\mu} Y_{h_{2}}^{\mu_{1}}+Y_{h_{1}}^{\mu_{1}} Y_{h_{2}}^{\mu}\right)}{\left(1-\left\langle M_{h_{1}}\right\rangle\right)\left(1-\left\langle M_{h_{2}}\right\rangle\right)}\left\langle\left(1-M_{h_{1}}\right)\left(1-M_{h_{2}}\right)\right\rangle \\
& -\sum_{p_{1} h_{1}} \frac{\left(Y_{h_{1}}^{\mu_{2}} X_{p_{1}}^{\mu^{\prime}}+Y_{h_{1}}^{\mu^{\prime}} X_{p_{1}}^{\mu_{2}}\right)\left(Y_{h_{1}}^{\mu_{1}} X_{p_{1}}^{\mu}+Y_{h_{1}}^{\mu} X_{p_{1}}^{\mu_{1}}\right)}{\left(1-\left\langle M_{p_{1}}\right\rangle\right)\left(1-\left\langle M_{h_{1}}\right\rangle\right)}\left\langle\left(1-M_{p_{1}}\right)\left(1-M_{h_{1}}\right)\right\rangle \\
& +\sum_{p_{1} p_{2}} \frac{X_{p_{1}}^{\mu_{2}} X_{p_{2}}^{\mu^{\prime}}\left(X_{p_{1}}^{\mu_{1}} X_{p_{2}}^{\mu}+X_{p_{1}}^{\mu} X_{p_{2}}^{\mu_{1}}\right)}{\left(1-\left\langle M_{p_{1}}\right\rangle\right)\left(1-\left\langle M_{p_{2}}\right\rangle\right)}\left\langle\left(1-M_{p_{1}}\right)\left(1-M_{p_{2}}\right)\right\rangle .
\end{aligned}
$$

\section{ACKNOWLEDGMENTS}

This work was supported in part by Conacyt (México), Conicet (Argentina), and by the DGES (Spain) under Contract BFM2000-1320-C02-02.

\section{REFERENCES}

1. B. D. Day, Rev. Mod. Phys. 39 (1967), 719; H. A. Bethe, Ann. Rev. Nucl. Part. Sci. 21 (1971), 93; J. P. Jeukenne, A. Lejeune, and C. Mahaux, Phys. Rep. 25 (1976), 83; A. D. Jackson, Ann. Rev. Nucl. Part. Sci. 33 (1983), 105.

2. S. Fantoni, Phys. Rev. B 29 (1984), 2544; K. E. Schmidt and V. R. Pandharipande, Phys. Lett. B 87 (1978), 11 ; A. D. Jackson, E. Krotschek, D. E. Metner, and R. A. Smith, Nucl. Phys. A 368 (1982), 125.

3. H. Kümmel, K. H. Lührmann, and J. B. Zabolitzky, Phys. Rep. 36 (1978), 1; R. F. Bishop and K. H. Lührmann, Phys. Rev. B 26 (1982), 5523.

4. J. P. Blaizot and G. Ripka, "Quantum Theory of Finite Systems," MIT Press, Cambridge, MA, 1986; P. Fulde, "Electron Correlations in Molecules and Solids," Springer Series in Solid-State Sciences, Springer-Verlag, Berlin/Heidelberg, 1991.

5. K. Hara, Prog. Theor. Phys. 32 (1964), 88.

6. D. J. Rowe, Rev. Mod. Phys. 40 (1968), 153; D. J. Rowe, Phys. Rev. 175 (1968), 1283; D. J. Rowe, "Nuclear Collective Motion," Methuen, London, 1970.

7. G. Röpke, Z. Phys. B 99 (1995), 83, and references therein.

8. T. Marumori, M. Yamamura, and A. Tokunaga, Prog. Theor. Phys. 31 (1964), 1009; T. Marumori, M. Yamamura, A. Tokunaga, and A. Takada, Prog. Theor. Phys. 32 (1964), 726.

9. P. Schuck, Z. Phys. 241 (1971), 395. 
10. P. Schuck and S. Ethofer, Nucl. Phys. A 212 (1973), 269.

11. J. Dukelsky and P. Schuck, Nucl. Phys. A 512 (1990), 466.

12. J. Dukelsky and P. Schuck, Phys. Lett. B 387 (1996), 233.

13. J. Dukelsky, G. Röpke, and P. Schuck, Nucl. Phys. A 628 (1998), 17.

14. J. Dukelsky and P. Schuck, Rev. Mex. Fis. Suppl. 244 (1998), 55.

15. J. Dukelsky and G. Sierra, Phys. Rev. Lett. 83 (1999), 172; G. Sierra et al., Phys. Rev. B 61 (2000), R11890.

16. J. Dukelsky, J. G. Hirsch, and P. Schuck, Eur. Phys. J. A 7 (2000), 155.

17. F. Catara, N. Dinh Dang, and M. Sambataro, Nucl. Phys. A 579 (1994), 1.

18. J. Toivanen and J. Suhonen, Phys. Rev. Lett. 75 (1995), 410.

19. F. Catara, G. Piccitto, M. Sambataro, and N. Van Giai, Phys. Rev. B 54 (1996), 17536.

20. R.W. Richardson, Phys. Rev. 141 (1966), 949.

21. J. Bang and J. Krumlinde, Nucl. Phys. 141 (1970), 18.

22. F. Braun and J. von Delft, Phys. Rev. Lett. 81 (1998), 4712.

23. M. Baranger, Phys. Rev. 120 (1960), 957.

24. P. Ring and P. Schuck, "The Nuclear Many Body Problem," Springer-Verlag, New York, 1980.

25. J. G. Hirsch, O. Civitarese, and M. Reboiro, Phys. Rev. C 60 (1999), 24309. 